RAIRO-Oper. Res. 55 (2021) S593-S624

https://doi.org/10.1051/ro/2019091

RAIRO Operations Research

www.rairo-ro.org

\title{
STATISTICAL TAYLOR SERIES EXPANSION: AN APPROACH FOR EPISTEMIC UNCERTAINTY PROPAGATION IN MARKOV RELIABILITY MODELS
}

\author{
Katia BaChI ${ }^{1, *}$, Karim AbBas ${ }^{1}$ And Bernd HeidergotT ${ }^{2}$
}

\begin{abstract}
In this paper we develop a new Taylor series expansion method for computing model output metrics under epistemic uncertainty in the model input parameters. Specifically, we compute the expected value and the variance of the stationary distribution associated with Markov reliability models. In the multi-parameter case, our approach allows to analyze the impact of correlation between the uncertainty on the individual parameters the model output metric. In addition, we also approximate true risk by using the Chebyshev' inequality. Numerical results are presented and compared to the corresponding Monte Carlo simulations ones.
\end{abstract}

Mathematics Subject Classification. 60J22, 41A58, 60K10.

Received November 5, 2018. Accepted September 18, 2019.

\section{INTRODUCTION}

Markovian models are frequently used to represent real and human-made systems such as industrial systems, power generation systems, and manufacturing systems, etc. Markov modeling techniques have been used by designers as a powerful and fundamental technique to analyze reliability, availability, maintainability, and safety of these systems $[29,30]$. The primary purpose of these models is to relate output metrics for a system to (some) input parameters. So, most frequently, it is assumed that these stochastic models are solved for fixed input parameters values. However, the input parameters are determined through statistical data (a finite number of observations), leading to uncertainty in the assessment of their values. This parametric uncertainty induced by the incomplete information concerning the parameter is called "epistemic uncertainty" [27]. Moreover, the input parameters are either physically controllable/adjustable or uncontrollable. For example, in a repairable system where system components are restored when they fail, the failure rate of a component is typically uncontrollable, whereas the repair rates or the maintenance policies are controllable. Uncontrollable parameters are only obtained through statistics. More formally, let $\theta$ be an uncontrollable parameter, and we assume the model input parameter $\theta$ is of the form

$$
\theta(\omega)=\bar{\theta}+\sigma \varepsilon(\omega)
$$

\footnotetext{
Keywords. Markov reliability model, epistemic uncertainty, correlation, risk analysis, fundamental matrix, Taylor series expansion, Monte Carlo simulation.

1 Research Unit LaMOS, Department of Operational Research, Faculty of Exact Sciences, University of Bejaia, Bejaia, Algeria.

2 Tinbergen Institute and Department Econometrics and Operations Research, VU University Amsterdam, Amsterdam, The Netherlands.

*Corresponding author: bachi.katia13@gmail.com
} 
where $\bar{\theta}$ is the predictor for $\theta, \sigma$ is the standard deviation of the same parameter, and $\varepsilon(\omega)$ is a random variable modeling the epistemic distribution. The parameters of the stochastic model which are not controllable may be considered to be random variables. In order to propagate the epistemic uncertainty on model input parameters, the model output needs to be related to the input parameters in a "functional form"; this functional relationship between the input parameters and the model output $Y$ can be expressed as

$$
Y(\omega)=g(\theta(\omega))
$$

where $g$ is the functional relationship.

The model in (1.1) together with (1.2) allows for different interpretations. Assuming that we have a test statistic $\theta(\omega)$ for $\theta$, we may think of $\sigma$ as the standard deviation of the statistic and $\varepsilon(\omega)$ as the noise of the data, which is typically standard normal distributed. Specifically, $\varepsilon(\omega)$ is a zero mean normal random variable and $\theta(\omega)$ is the actual (unknown) value of the interest parameter. Increasing the sample size effects in this model $\sigma$ and our formulas allows tracing the impact of larger sample sizes on the reduction of the uncertainty in output. Alternatively, $\bar{\theta}$ may be obtained from expert knowledge and $\varepsilon(\omega)$ expresses the epistemic insecurity in the expert knowledge, where $\sigma$ serves as scaling parameter. The epistemic distribution is typically obtained by identifying the distribution that, given the expert knowledge available, maximizes the entropy.

The objective of this paper is to develop a new approach based on Taylor series expansion for propagating uncertainty in input-output models. For that, we develop the model output $Y$ into a Taylor series with respect to the input parameters and then make use of the properties of expectation and variance for computing the expectation and variance of reliability indices. We show that sensitivity analysis and statistical estimation can be integrated into one framework. Specifically, by using this new approach, we establish an approximative expression for the components of the stationary distribution of the Markov chain describing the state of the considered model. These components are represented as polynomial functions of the input parameters, which are given under the form (1.1). More specifically, we provide a recursive form of the higher derivatives of the stationary distribution in terms of the fundamental matrix of the associated continuous-time Markov chain [20]. In addition, we establish an expression for the remainder term of the Taylor series, and we include the effect of the correlation between the input parameters, which are considered as uncertain random variables. Furthermore, we use the Chebyshev's inequality for estimating the risk incurred by working with uncertain performance measures rather than those evaluated at fixed parameters. We also use another uncertainty analysis approach based on Monte Carlo simulation. Exploiting properties and transformations of expectation and variance, we numerically compute the expected value and the variance of the stationary distribution associated with the studied Markov reliability models.

Analytic methods for parametric epistemic uncertainty propagation in Markov models have been proposed in literature before; for references, see e.g., $[4,6-9,34,36]$. Most of these approaches are based on Taylor series expansion, where their coefficients are given in terms of the group inverse of the underlying Markov chain [17]. Unfortunately, no efficiently computable form of the remainder the considered Taylor series are provided in the aforementioned references. The approach developed in this paper overcomes this drawback and provides efficient bounds on the remainder term corresponding to the Taylor series. Our new computational approach is based on Taylor series expansion for continuous-time Markov chains, for which the Taylor series coefficients are obtained in terms of the fundamental matrix $Z$ of the underlying Markov chain, as well as we provide an efficient way with the potential of computing the remainder term of the Taylor series. It is worth noting that several computational techniques for finding $Z$ have been proposed in the literature, see e.g., $[2,12-14,22,24,33]$. We also note that our framework is different from the one proposed in [1,10], where the higher-order sensitivity of the Markov chain stationary distribution with respect to model parameters is expressed in terms of the deviation matrix, see e.g., $[5,16,23]$, and these results have been obtained for fixed parameter values.

In this paper we propose a new framework involving the use of multivariate Taylor series expansions to propagate epistemic uncertainty to reliability indices in Markov reliability models. The remainder of this paper is organized as follows. In Section 2, we establish the higher-order sensitivity of the continuous-time Markov chain stationary distribution with respect to a single-parameter. The generalization of these results to the case 
of the higher-order sensitivity with respect to a multiple-parameter is given in Section 4. Markov reliability models are analyzed in Sections 3 and 5. Finally, Section 7 summarizes the investigation done and draws the conclusions.

\section{The SINGLE-PARAMETER CASE}

\subsection{Higher-order derivative expressions}

In this section, we establish a closed-form expression for the higher-order sensitivity of the stationary distribution of a continuous-time Markov chain with respect to a single parameter.

Let $\mathcal{X}=\left\{X_{t}: t \geq 0\right\}$ be a time-homogeneous, continuous-time ergodic Markov chain on a finite state space $S=\{0,1,2, \ldots, N-1\}$. Throughout this paper we will denote the generator matrix of $\mathcal{X}$ by $Q=\left(q_{i j}\right)_{i, j \in S}$, where for $i \neq j$, the components $q_{i j}$ are non-negative and the components $q_{i i}$ are chosen such that $\sum_{j \in S} q_{i j}=0$, for all $i \in S$. Let $e=(1,1, \ldots, 1)^{T}$ be the unit vector, where the superscript $T$ denotes the transpose. We suppose that $\mathcal{X}$ has a unique stationary distribution, denoted by $\pi=(\pi(0), \pi(1), \ldots, \pi(N-1))$, solving $\pi Q=0$ and $\pi e=1$. Assume that $Q$ depends on certain parameter $\theta$; then $Q$ may be considered a mapping of $\theta$, in writing, $Q_{\theta}$. In this section, we are mainly interested in the effect of a change in $\theta$ on the stationary distribution. More formally, let $\pi_{\theta}$ denote the stationary distribution associated with $Q_{\theta}$, then, we seek to derive a formula for the $n$ th-order derivative of the stationary distribution $\mathrm{d}^{n} \pi_{\theta} / \mathrm{d} \theta^{n}$. Our approach has an underlying assumption that only the components of the generator matrix $Q_{\theta}$ are differentiable functions with respect to the parameter $\theta$. Specifically, we will express this derivative in closed form as function of the fundamental matrix of the underlying Markov chain $\mathcal{X}$; it is denoted by $Z_{\theta}$, and is defined as follows [21]:

$$
Z_{\theta} \triangleq\left(\Pi_{\theta}-Q_{\theta}\right)^{-1}
$$

where $\Pi_{\theta}=e \pi_{\theta}$. The definition of this matrix for the continuous-time Markov chain with finite state space was introduced by Kemeny and Snell in [21]. It is worth noting that the existence of $Z_{\theta}$ is assured by the fact that $S$ is finite.

In the following theorem we will present a recursive formula for the higher-order derivatives of $\pi_{\theta}$, with respect to $\theta$ in terms of the fundamental matrix $Z_{\theta}$. With these derivatives at hand, we develop a framework which will be used afterward in our analysis.

Theorem 2.1. Assume that the generator matrix $Q_{\theta}$ is element-wise n-times differentiable at $\theta \in \Theta$, where $\Theta$ is a closed interval. Provided that

$$
\frac{1}{|\Delta|}\left|Q_{\theta+\Delta}^{(m)}(i, j)-Q_{\theta}^{(m)}(i, j)\right| \leq K_{m}
$$

for $0 \leq m<n$, the $n$ th-order derivative of the stationary distribution is given as follows

$$
\frac{\mathrm{d}^{n} \pi_{\theta}}{\mathrm{d} \theta^{n}}=\pi_{\theta}^{(n)}=\sum_{m=0}^{n-1}\left(\begin{array}{c}
n \\
m
\end{array}\right) \pi_{\theta}^{(m)} Q_{\theta}^{(n-m)} Z_{\theta},
$$

where $Q_{\theta}^{(k)}$ is the matrix of the element-wise kth-order derivative of the generator matrix $Q_{\theta}$ with respect to $\theta$.

Proof. We prove (2.2) by induction on $n$. Differentiability of $\pi_{\theta}$ with respect to $\theta$ is established in [11]. For $n=1$ we check that

$$
\pi_{\theta}^{\prime}=\pi_{\theta} Q_{\theta}^{\prime} Z_{\theta}
$$

For any continuous-time Markov chain with generator matrix $Q_{\theta}$, we have

$$
\left\{\begin{array}{l}
\pi_{\theta} Q_{\theta}=0 \\
\pi_{\theta} e=1
\end{array}\right.
$$


Taking the derivative of both sides of the equation (2.3) with respect to $\theta$, we get

$$
\left\{\begin{array}{l}
\pi_{\theta}^{\prime} Q_{\theta}+\pi_{\theta} Q_{\theta}^{\prime}=0 \\
\pi_{\theta}^{\prime} e=0
\end{array}\right.
$$

Multiplying both sides of the second equation in (2.4) on the right by $\pi_{\theta}$, we obtain

$$
\pi_{\theta}^{\prime} \Pi_{\theta}=0 .
$$

Inserting (2.5) in the left-hand side of the first equation in (2.4), we have

$$
\pi_{\theta}^{\prime} Q_{\theta}-\underbrace{\pi_{\theta}^{\prime} \Pi_{\theta}}_{=0}+\pi_{\theta} Q_{\theta}^{\prime}=0 .
$$

Then, it holds that

$$
\pi_{\theta}^{\prime}\left(\Pi_{\theta}-Q_{\theta}\right)=\pi_{\theta} Q_{\theta}^{\prime} .
$$

Since the Markov chain associated with $Q_{\theta}$ is ergodic, the matrix $\left(\Pi_{\theta}-Q_{\theta}\right)$ is invertible. It holds that

$$
\pi_{\theta}^{\prime}=\pi_{\theta} Q_{\theta}^{\prime} \underbrace{\left(\Pi_{\theta}-Q_{\theta}\right)^{-1}}_{=Z_{\theta}} .
$$

Thus, it has been shown that the statement (2.2) is true for $n=1$.

Assume that (2.2) is correct for some positive integer $k$. Then, we have

$$
\pi_{\theta}^{(k)}=\sum_{m=0}^{k-1}\left(\begin{array}{c}
k \\
m
\end{array}\right) \pi_{\theta}^{(m)} Q_{\theta}^{(k-m)} Z_{\theta} .
$$

Multiplying both sides of the equation (2.6) on the right by $\left(\Pi_{\theta}-Q_{\theta}\right)$, we get

$$
\pi_{\theta}^{(k)}\left(\Pi_{\theta}-Q_{\theta}\right)=\sum_{m=0}^{k-1}\left(\begin{array}{c}
k \\
m
\end{array}\right) \pi_{\theta}^{(m)} Q_{\theta}^{(k-m)} .
$$

On other hand, repeating to take derivatives of both sides of the second equation in (2.4), we get for any $k \geq 1$

$$
\pi_{\theta}^{(k)} e=0
$$

which implies that

$$
\pi_{\theta}^{(k)} \Pi_{\theta}=0 .
$$

This together with the equation (2.7) allows to obtain

$$
-\pi_{\theta}^{(k)} Q_{\theta}=\sum_{m=0}^{k-1}\left(\begin{array}{c}
k \\
m
\end{array}\right) \pi_{\theta}^{(m)} Q_{\theta}^{(k-m)} .
$$

Taking again derivatives of both sides of equation (2.8) with respect to $\theta$, then we get

$$
-\pi_{\theta}^{(k+1)} Q_{\theta}-\pi_{\theta}^{(k)} Q_{\theta}^{\prime}=\sum_{m=0}^{k-1}\left(\begin{array}{c}
k \\
m
\end{array}\right)\left[\pi_{\theta}^{(m+1)} Q_{\theta}^{(k-m)}+\pi_{\theta}^{(m)} Q_{\theta}^{(k-m+1)}\right] .
$$

By computation, we arrive at

$$
\pi_{\theta}^{(k+1)}=\sum_{m=0}^{k}\left(\begin{array}{c}
k+1 \\
m
\end{array}\right) \pi_{\theta}^{(m)} Q^{(k-m+1)} Z_{\theta} .
$$

By induction, the statement (2.2) holds for all positive integer $n$. 
Remark 2.2. Note that our approach is slightly different of the one introduced in [28] and that our expression for the higher-order sensitivity of stationary distribution to single-parameter is comparable to the one given by (1.2) therein. In fact, the expression (2.2) is the analogous formula for discrete-time Markov chains given in [28]. If $Q_{\theta}$ is a generator matrix of some uniformizable Markov chain with rate $\gamma$ (i.e., $\gamma=\sup _{i}\left|q_{i i}\right|<\infty$ ), then its transition matrix is given by

$$
P_{\gamma}=I+\frac{1}{\gamma} Q
$$

Inserting (2.9) into (2) given in [28] yields the alternative formula (2.2).

Remark 2.3. Due to the fact that the components of the generator matrix $Q_{\theta}$ are generally linear in $\theta$, hence for all $m \geq 2, q_{i j}^{(m)}=0$. Revisiting the proposed formula (2.2), by taking into account this, one can obtain

$$
\pi_{\theta}^{(n)}=n ! \pi_{\theta}\left(Q_{\theta}^{\prime} Z_{\theta}\right)^{n}
$$

Note that formula (2.2) given in Theorem 2.1 is significantly more difficult than the above one (2.10) as in the case of a non-linear form of components of $\pi_{\theta}$ all their higher-order derivatives maybe different from zero.

Moreover, an equivalent formula of (2.10) for the first derivative of the stationary distribution of a continuoustime Markov process with denumerable state has been obtained by Heidergott et al. [11] in terms of the deviation matrix. However, the authors in [11] have followed a different line of the proof, where they have supposed that the infinitesimal generator associated with the Markov process satisfies the Lipschitz condition.

Example 2.4. Using formula (2.10), one can easily obtain an explicit expression for the lower derivatives of $\pi_{\theta}$ as follows

$$
\pi_{\theta}^{\prime}=\pi_{\theta} Q_{\theta}^{\prime} Z_{\theta}, \pi_{\theta}^{(2)}=2 \pi_{\theta}\left(Q_{\theta}^{\prime} Z_{\theta}\right)^{2}, \quad \text { and } \quad \pi_{\theta}^{(3)}=6 \pi_{\theta}\left(Q_{\theta}^{\prime} Z_{\theta}\right)^{3} .
$$

\subsection{Single-valued statistical Taylor series expansion}

Using the higher-order sensitivity of stationary distribution with respect to the parameter $\theta(\omega)$ introduced in (2.2), one can propagate the epistemic uncertainty of model input parameters to the output measure $\pi(\omega)$. Under the conditions put forward in Theorem 2.1 it holds that $\pi(\omega)$ can be developed into the following Taylor series

$$
\pi(\omega) \triangleq \pi_{\bar{\theta}+\sigma \varepsilon(\omega)}=\underbrace{\pi_{\bar{\theta}}}_{\text {Value at fixed parameter }}+\underbrace{\sum_{n=1}^{\infty} \frac{(\theta(\omega)-\bar{\theta})^{n}}{n !} \pi_{\bar{\theta}}^{(n)}}_{\text {Value with uncertainty }},
$$

where $\pi_{\bar{\theta}}^{(n)}$ denotes the $n$th order derivative of $\pi_{\theta}$ with respect to parameter $\theta$ evaluated at $\bar{\theta}$. Note that the derivative of order zero of $\pi_{\theta}$ is $\pi_{\theta}$ itself. In the following we refer to the Taylor series in (2.11) as statistical Taylor series.

In the case where the entries of $Q_{\theta}$ are linear mappings of $\theta$, using the representation of the higher-order derivatives of the stationary distribution given in (2.10), the series expansion given in (2.11) can be written in the more compact form as

$$
\pi_{\bar{\theta}+\sigma \varepsilon(\omega)}=\pi_{\bar{\theta}} \sum_{n=0}^{\infty}\left((\theta(\omega)-\bar{\theta}) Q_{\bar{\theta}}^{\prime} Z_{\bar{\theta}}\right)^{n} .
$$

Using the approach outlined above, one can easily numerically compute the different moments of the considered output measure. In particular, assuming that the moments $\mathbb{E}\left[(\varepsilon(\omega))^{n}\right]$ are finite, the expected value and the variance of the the stationary distribution $\pi(\omega)$ can be calculated as follows

$$
\mathbb{E}[\pi(\omega)]=\sum_{n=0}^{\infty} \frac{\pi_{\bar{\theta}}^{(n)}}{n !} \mathbb{E}\left[(\theta(\omega)-\bar{\theta})^{n}\right]=\sum_{n=0}^{\infty} \frac{\sigma^{n} \pi_{\bar{\theta}}^{(n)}}{n !} \mathbb{E}\left[(\varepsilon(\omega))^{n}\right],
$$


and

$$
\operatorname{Var}[\pi(\omega)] \triangleq \mathbb{E}\left[(\pi(\omega)-\bar{\pi}(\omega))^{2}\right]=\mathbb{E}\left[(\pi(\omega))^{2}\right]-\mathbb{E}[\bar{\pi}(\omega)]^{2} .
$$

Example 2.5. If we assume that in the uncertain input parameter $\theta(\omega)$, see (1.1), the random variable modeling the epistemic uncertainty distribution $\varepsilon(\omega)$ is described by a standard normal distribution denoted by $\mathcal{N}(0,1)$. Then, the $n$th moments exist and are finite for any non-negative integer $n \geq 1$. We have

$$
\mathbb{E}\left[(\varepsilon(\omega))^{n}\right]= \begin{cases}0, & \text { if } n \text { is odd } \\ (n-1) ! !, & \text { if } n \text { is even, }\end{cases}
$$

where $(n-1) !$ ! is the semifactorial of $(n-1)$ which is defined as

$$
(n-1) ! !=\prod_{l=1}^{s}(2 l-1),
$$

and $s=\lceil n / 2\rceil$, with $\lceil x\rceil$ is the smallest integer larger of equal to $x$.

Specifically, for an even positive integer $n=2 p, p \geq 0$, the expected value of $\pi(\omega)$ given in (2.13), can be obtained in more explicit form as follows

$$
\begin{aligned}
\mathbb{E}[\pi(\omega)] & =\sum_{n=0}^{\infty} \frac{\sigma^{n} \pi_{\bar{\theta}}^{(n)}}{n !} \mathbb{E}\left[(\varepsilon(\omega))^{n}\right] \\
& =\pi_{\bar{\theta}} \sum_{p=0}^{\infty}\left(\sigma Q_{\bar{\theta}}^{\prime} Z_{\bar{\theta}}\right)^{2 p}(2 p-1) ! !
\end{aligned}
$$

In the same vein, the variance of $\pi_{i}(\omega)$ can be derived as follows

$$
\begin{aligned}
\operatorname{Var}\left[\pi_{i}(\omega)\right]= & \sum_{n=1}^{\infty} \sum_{m=1}^{\infty} \alpha_{i, n} \alpha_{i, m} \operatorname{Cov}\left(\varepsilon(\omega)^{n}, \varepsilon(\omega)^{m}\right) \\
= & \sum_{n=1}^{\infty} \sum_{m=1}^{\infty} \alpha_{i, n} \alpha_{i, m}\left[\mathbb{E}\left(\varepsilon(\omega)^{n+m}\right)-\mathbb{E}\left(\varepsilon(\omega)^{n}\right) \mathbb{E}\left(\varepsilon(\omega)^{m}\right)\right] \\
= & \sum_{p=1}^{\infty} \sum_{q=1}^{\infty} \alpha_{i, 2 p} \alpha_{i, 2 q}\left[\mathbb{E}\left(\varepsilon(\omega)^{2 p+2 q}\right)-\mathbb{E}(\varepsilon(\omega))^{2 p} \mathbb{E}(\varepsilon(\omega))^{2 q}\right] \\
& +\sum_{p=0}^{\infty} \sum_{q=0}^{\infty} \alpha_{i, 2 p+1} \alpha_{i, 2 q+1} \mathbb{E}\left(\varepsilon(\omega)^{2 p+2 q+2}\right) \\
= & \sum_{p=1}^{\infty} \sum_{q=1}^{\infty} \alpha_{i, 2 p} \alpha_{i, 2 q}[(2 p+2 q-1) ! !-(2 p-1) ! !(2 q-1) ! !] \\
& +\sum_{p=0}^{\infty} \sum_{q=0}^{\infty} \alpha_{i, 2 p+1} \alpha_{i, 2 q+1}(2 p+2 q+1) ! !
\end{aligned}
$$

where

$$
\alpha_{i, k}=\frac{\sigma^{k}}{k !} \pi_{\bar{\theta}}^{(k)}=\pi_{\bar{\theta}}\left(\sigma Q_{\bar{\theta}}^{\prime} Z_{\bar{\theta}}\right)^{k} e_{i}^{T}
$$

with $e_{i}^{T}=(0 \ldots, \underbrace{1}_{\text {at the } i \text { position }}, \ldots 0)$. 
Introduce the $k$ th order Taylor approximation of $\pi(\omega)$ at $\bar{\theta}$ defined as follows

$$
T_{\theta}(k, \varepsilon(\omega))=\sum_{n=0}^{k} \frac{(\theta(\omega)-\bar{\theta})^{n}}{n !} \pi_{\bar{\theta}}^{(n)} .
$$

The remainder term is given by

$$
R_{\theta}(k, \varepsilon(\omega))=\pi(\omega)-T_{\theta}(k, \varepsilon(\omega)) .
$$

Example 2.6. For the normal distributed noise model presented in Example 2.5, the expected value and variance of $\pi(\omega)$ at the truncated Taylor series are given respectively by

$$
\begin{aligned}
\mathbb{E}\left[T_{\theta}(k, \varepsilon(\omega))\right]= & \pi_{\bar{\theta}} \sum_{p=0}^{\lceil k / 2\rceil}\left(\sigma Q_{\bar{\theta}}^{\prime} Z_{\bar{\theta}}\right)^{2 p}(2 p-1) ! ! \\
\operatorname{Var}\left[T_{\theta}(k, \varepsilon(\omega))_{i}\right]= & \sum_{p=1}^{\lceil k / 2\rceil} \sum_{q=1}^{\lceil k / 2\rceil} \alpha_{i, 2 p} \alpha_{i, 2 q}[(2 p+2 q-1) ! !-(2 p-1) ! !(2 q-1) ! !] \\
& +\sum_{p=0}^{\lceil k-1 / 2\rceil} \sum_{q=0}^{\lceil k-1 / 2\rceil} \alpha_{i, 2 p+1} \alpha_{i, 2 q+1}(2 p+2 q+1) ! !
\end{aligned}
$$

Remark 2.7. In practice, both parameters $\bar{\theta}$ (the estimated mean value of the random variable $\theta$ ) and $\sigma$ (the standard deviation of $\theta$ ) are commonly determined either from a finite number of observations (data) or are based on experts' predictions. Hence, these parameters can be computed using any standard statistical technique.

\subsection{Bounds for the statistical remainder term}

To use the Taylor series expansion introduced above in (2.11), it is mandatory to consider its truncated version. Therefore, in practice, we limit to use just a finite number of first terms of the Taylor series. For practical purposes, one needs to give a quantitative bound for the remainder term. For that, the error introduced by this truncation or approximation (see (2.17)) can be expressed by the Peano form of the remainder as follows

$$
R_{\theta}(k, \varepsilon(\omega))=(\theta(\omega)-\bar{\theta})^{k} \phi_{k}(\theta(\omega)),
$$

where $\lim _{\theta(\omega) \rightarrow \bar{\theta}} \phi_{k}(\theta(\omega))=0$.

Under the assumption of analyticity of the stationary distribution $\pi_{\bar{\theta}}$ (i.e., it is infinitely differentiable with respect to $\theta$ ), a typical form for the function $\phi_{k}$ can be chosen as follows

$$
\phi_{k}(\theta(\omega))=\sum_{n=k+1}^{\infty} \frac{(\theta(\omega)-\bar{\theta})^{n-k}}{n !} \pi_{\bar{\theta}}^{(n)} .
$$

For computational purposes, one needs to estimate the following remainder term

$$
R_{\theta}(k, \varepsilon(\omega))=(\theta(\omega)-\bar{\theta})^{k} \sum_{n=k+1}^{\infty} \frac{(\theta(\omega)-\bar{\theta})^{n-k}}{n !} \pi_{\bar{\theta}}^{(n)} .
$$

This idea is already outlined in [1] for the estimate of the Lagrange form of the remainder, where it is encountered the problem of the estimate of the higher derivative of the stationary distribution of $\pi$ at $\theta$; however, in this paper, we use another form (Peano form) for the remainder term, that is slightly different from 
the Lagrange one, and for which the problem mentioned above is not posed. Note also that in [1] the sensitivity of the stationary distribution is expressed in terms of the deviation matrix associated with the Markov chain, but here it is expressed in function of the fundamental matrix of the corresponding Markov chain. Moreover, in the present paper, we deal with the sensitivity of continuous-time Markov chain, so as indicated above that, in this case, the components of the generator matrix $Q_{\theta}$ are linear mappings of $\theta$. In the following, we develop a new approach allowing us to derive an explicit expression for the remainder term under Peano form.

$$
\begin{aligned}
R_{\theta}(k, \varepsilon(\omega)) & =(\theta(\omega)-\bar{\theta})^{k} \sum_{n=k+1}^{\infty} \frac{(\theta(\omega)-\bar{\theta})^{n-k}}{n !} \pi_{\bar{\theta}}^{(n)} \\
& =\pi_{\bar{\theta}}(\theta(\omega)-\bar{\theta})^{k} \sum_{n=k+1}^{\infty}(\theta(\omega)-\bar{\theta})^{n-k}\left(Q_{\bar{\theta}}^{\prime} Z_{\bar{\theta}}\right)^{n} \\
& =\pi_{\bar{\theta}} \sum_{n=k+1}^{\infty}\left((\theta(\omega)-\bar{\theta}) Q_{\bar{\theta}}^{\prime} Z_{\bar{\theta}}\right)^{n} .
\end{aligned}
$$

In order to obtain a useful estimate for the remainder term, we will use the expected of the remainder term established in (2.19). For that, we first estimate the expectation of $R_{\theta}(k, \varepsilon(\omega))$. This estimate is given in the Lemma below.

Lemma 2.8. Under (1.1) with $\varepsilon$ standard normal distributed, the expected value of the remainder term is given as follows

$$
\mathbb{E}\left(R_{\theta}(k, \varepsilon(\omega))\right) \leq \pi_{\bar{\theta}} \sum_{p=\lceil k / 2\rceil+1}^{\infty}\left(2 \sigma Q_{\bar{\theta}}^{\prime} Z_{\bar{\theta}}\right)^{2 p} .
$$

Proof. By definition, the expected value of the remainder term can be computed as follows

$$
\begin{aligned}
\mathbb{E}\left(R_{\theta}(k, \varepsilon(\omega))\right) & =\pi_{\bar{\theta}} \sum_{n=k+1}^{\infty} \mathbb{E}(\theta(\omega)-\bar{\theta})^{n}\left(Q_{\bar{\theta}}^{\prime} Z_{\bar{\theta}}\right)^{n} \\
& =\pi_{\bar{\theta}} \sum_{p=\lceil k / 2\rceil+1}^{\infty} \frac{(2 p) !}{2^{p} p !}\left(\sigma Q_{\bar{\theta}}^{\prime} Z_{\bar{\theta}}\right)^{2 p} .
\end{aligned}
$$

Using the fact that for any positive integer $p$, we have

$$
(2 p) ! \leq 2^{3 p} p !
$$

Noting that the inequality (2.21) can be easily proven by induction on $p$. Hence, it follows that

$$
\begin{aligned}
\mathbb{E}\left(R_{\theta}(k, \varepsilon(\omega))\right) & \leq \pi_{\bar{\theta}} \sum_{p=\lceil k / 2\rceil+1}^{\infty} \frac{2^{3 p} p !}{2^{p} p !}\left(\sigma Q_{\bar{\theta}}^{\prime} Z_{\bar{\theta}}\right)^{2 p} \\
& \leq \pi_{\bar{\theta}} \sum_{p=\lceil k / 2\rceil+1}^{\infty} 2^{2 p}\left(\sigma Q_{\bar{\theta}}^{\prime} Z_{\bar{\theta}}\right)^{2 p} \\
& \leq \pi_{\bar{\theta}} \sum_{p=\lceil k / 2\rceil+1}^{\infty}\left(2 \sigma Q_{\bar{\theta}}^{\prime} Z_{\bar{\theta}}\right)^{2 p}
\end{aligned}
$$

which proves the claim. 
In order to estimate the remainder term, we will use the 1-norm of a vector $v$ (defined as the absolute entry sum), denoted by $\|v\|_{1}$, and the $\infty$-norm of a matrix $M$ (defined as its maximum absolute row sum), denoted by $\|M\|_{\infty}$.

Theorem 2.9. Assume (1.1) with $\varepsilon$ standard normal distributed and let the conditions put forward in Theorem 2.1 be satisfied. If the following condition holds:

(C) there exists a finite constant $c$ such that

$$
\left\|\left(Q_{\bar{\theta}}^{\prime} Z_{\bar{\theta}}\right)^{2}\right\| \leq c<1 / 4 \sigma^{2}
$$

then

$$
\left\|\mathbb{E}\left(R_{\theta}(k, \varepsilon(\omega))\right)\right\| \leq \frac{\left(4 \sigma^{2} c\right)^{\lceil k / 2\rceil+1}}{1-4 \sigma^{2} c} .
$$

Proof. Taking $\|.\|_{\infty}$ norm on both sides of (2.20) yields

$$
\begin{aligned}
\left\|\mathbb{E}\left(R_{\theta}(k, \varepsilon(\omega))\right)\right\| & \leq\left\|\pi_{\bar{\theta}} \sum_{p=\lceil k / 2\rceil+1}^{\infty}\left(2 \sigma Q_{\bar{\theta}}^{\prime} Z_{\bar{\theta}}\right)^{2 p}\right\| \\
& \leq\left\|\pi_{\bar{\theta}}\right\| \sum_{p=\lceil k / 2\rceil+1}^{\infty}\left(4 \sigma^{2}\left\|\left(Q_{\bar{\theta}}^{\prime} Z_{\bar{\theta}}\right)^{2}\right\|\right)^{p} .
\end{aligned}
$$

By (C) it holds that

$$
\begin{aligned}
\left\|\mathbb{E}\left(R_{\theta}(k, \varepsilon(\omega))\right)\right\| & \leq \sum_{p=\lceil k / 2\rceil+1}^{\infty}\left(4 \sigma^{2} c\right)^{p} \\
& \leq \frac{\left(4 \sigma^{2} c\right)^{\lceil k / 2\rceil+1}}{1-4 \sigma^{2} c} .
\end{aligned}
$$

Subsequently, a detailed discussion on the convergence of the series in (2.12) will be outlined in the next subsection.

\subsection{Convergence of the statistical Taylor series}

Frequently, the radius of convergence $r$ of the power series (2.12) is given by the Cauchy-Hadamard formula

$$
1 / r=\lim \sup _{n \rightarrow \infty}\left\|\frac{\pi^{(n)}}{n !}\right\|^{1 / n} .
$$

An upper bound for $1 / r$ is provided in the following theorem.

Theorem 2.10. Assume (1.1) with $\varepsilon$ standard normal distributed. Under the conditions of Theorem 2.1 and provided that condition $\mathbf{( C )}$ holds, we have

$$
1 / r<1 / 2 \sigma
$$

where $\sigma$ is the standard deviation of the parameter $\theta(\omega)$. 
Proof. From (2.23), it follows that

$$
\begin{aligned}
1 / r & \leq\left\|\pi_{\bar{\theta}}\left(Q_{\bar{\theta}}^{\prime} Z_{\bar{\theta}}\right)\right\| \\
& \leq\left\|Q_{\bar{\theta}}^{\prime} Z_{\bar{\theta}}\right\| .
\end{aligned}
$$

By condition (C),

$$
\left\|Q_{\bar{\theta}}^{\prime} Z_{\bar{\theta}}\right\|^{2}<1 / 4 \sigma^{2}
$$

and we arrive at

$$
1 / r^{2}<1 / 4 \sigma^{2}
$$

Hence, we get

$$
r>2 \sigma .
$$

On the other hand, the series (2.12) converges if

$$
|\theta(\omega)-\bar{\theta}|<r
$$

which implies that

$$
|\theta(\omega)-\bar{\theta}|^{2 p}<r^{2 p}
$$

Taking the expectation on both sides of (2.26) yields

$$
\mathbb{E}\left(|\theta(\omega)-\bar{\theta}|^{2 p}\right)=\sigma^{2 p} \frac{(2 p) !}{2^{p} p !}<r^{2 p} .
$$

Using (2.21), we obtain

$$
\mathbb{E}\left(|\theta(\omega)-\bar{\theta}|^{2 p}\right) \leq(2 \sigma)^{2 p}<r^{2 p}
$$

This allows us to get

$$
2 \sigma<r
$$

From (2.25) and (2.27) we get the following upper bound

$$
1 / r<1 / 2 \sigma
$$

\subsection{Chebyshev risk bounds}

Let $f \geq 0$ be a cost function. The risk in taking $\pi_{\bar{\theta}} f$ as cost measure rather than $\pi(\omega) f$ is given by

$$
\pi(\omega) f-\pi_{\bar{\theta}} f
$$

where the decision maker wants to avoid large positive values of the above expression as this means that the actual costs $\pi(\omega) f$ were significantly larger than the projected costs $\pi_{\bar{\theta}} f$. In order to asses the risk incurred by working with $\pi(\omega) f$ one wants to bound

$$
P\left(\pi(\omega) f-\pi_{\bar{\theta}} f \geq \eta\right),
$$

where $\eta$ is a problem dependent threshold expressing the deviation from the planned the decision maker is willing to take. In this paper we consider models in which $\pi_{\bar{\theta}} f$ can be obtained by closed form solution.

We analyze the risk that the actual performance deviates more than, say, $r$ percent from the numerical value $\pi_{\bar{\theta}} f$. For this we let

$$
\eta=\frac{r}{100} \pi_{\bar{\theta}} f
$$


and

$$
R_{\theta} f=\sum_{n=1}^{\infty} \frac{\mathbb{E}\left[\varepsilon(\omega)^{n}\right]}{n !} \sigma^{n} \pi_{\bar{\theta}}^{(n)} f
$$

Then it follows from (2.11) that

$$
\mathbb{E}[\pi(\omega) f]=\mathbb{E}\left[\pi_{\bar{\theta}+\sigma \varepsilon(\omega)} f\right]=\pi_{\bar{\theta}} f+R_{\theta} f,
$$

which implies

$$
|\pi(\omega) f-\mathbb{E}[\pi(\omega) f]| \geq\left|\pi(\omega) f-\pi_{\bar{\theta}} f\right|-\left|R_{\theta} f\right|,
$$

and

$$
\begin{aligned}
P\left(\left|\pi(\omega) f-\pi_{\bar{\theta}} f\right| \geq \eta\right) & =P\left(\left|\pi(\omega) f-\mathbb{E}\left[\pi(\omega) f+R_{\theta} f\right]\right| \geq \eta\right) \\
& \leq P\left(|\pi(\omega) f-\mathbb{E}[\pi(\omega) f]| \geq \eta-\left|R_{\theta} f\right|\right) .
\end{aligned}
$$

We let $\delta=\eta-\left|R_{\theta} f\right|>0$, and applying Chebyshev's inequality to (2.29), we get

$$
P(|\pi(\omega) f-\mathbb{E}[\pi(\omega) f]| \geq \delta) \leq \frac{\operatorname{Var}[\pi(\omega) f]}{\delta^{2}} .
$$

To summarize, we arrive at

$$
P\left(\left|\pi(\omega) f-\pi_{\bar{\theta}} f\right| \geq \eta\right) \leq \frac{\mathbb{V a r}[\pi(\omega) f]}{\left(\frac{r}{100} \pi_{\bar{\theta}} f-\left|\mathbb{E}[\pi(\omega) f]-\pi_{\bar{\theta}} f\right|\right)^{2}} .
$$

\section{Single-VAlue: Extended nUmerical EXAMPle}

By the Taylor series expansion approach for Markov chains a wide class of reliability models can be systematically and thoroughly studied. This approach leads in many cases to numerically stable algorithms for stationary Markov chain. In this section, we use this approach to numerically analyze the parametric epistemic uncertainty propagation in certain Markov reliability models. Particularly, we discuss two kinds of systems: a redundancy-standby system and a Jackson network with group breakdowns and repairs, where we consider the perturbation of a single parameter.

\subsection{Redundancy-standby system}

Components or subsystems in a system may be more relevant for the system's reliability than others. In order to improve the reliability and availability of a system, one may either use components with high reliability or redundancy-standby systems. In this section, we consider a simple standby model; see Figure 1. The system has two components in standby configuration, one online and other as a backup. When a primary component fails after some time that has an exponential distribution with parameter $\lambda$, standby component is started up. There is a repair facility in which the repair time is exponentially distributed with parameter $\mu$. The infinitesimal generator associated with the Markov process, representing the number of operating components, is given by

$$
Q_{\lambda}=\left(\begin{array}{ccc}
-\mu & \mu & 0 \\
\lambda & -(\lambda+\mu) & \mu \\
0 & \lambda & -\lambda
\end{array}\right) .
$$

Solving system, see (2.3), we obtain

$$
\pi_{i}=\frac{(\mu / \lambda)^{i}}{1+(\mu / \lambda)+(\mu / \lambda)^{2}}, \quad \text { for } i=0,1,2 .
$$




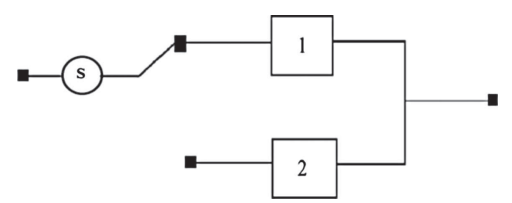

Figure 1. Two components standby system.

In the sequel, we assume that the failure rate $\lambda$ is determined from a finite number of observations and has uncertainty associated with it. Using the approach outlined in Section 2.2, we will numerically calculate the expectation and the variance of the stationary distribution corresponding to the studied model. In this regard, a new model is introduced now for the failure rate

$$
\lambda=\bar{\lambda}+\sigma \varepsilon,
$$

with $\varepsilon$ standard normal distributed, and moreover $\bar{\lambda}$ is the estimated mean value provided by the statistic of the failure rate $\lambda, \sigma$ its standard deviation and $\varepsilon$ is a random variable modeling the epistemic distribution, which follows the standard normal distribution.

The fundamental matrix $Z_{\lambda}$ of the continuous-time Markov chain is given by

$$
\begin{aligned}
& Z_{\lambda}= \\
& \left(\begin{array}{ccc}
\frac{\mu^{2}\left(\lambda^{2}+3 \lambda\right)+\mu\left(\lambda^{3}+\lambda^{2}\right)+\lambda^{4}+\mu^{3}}{\left(\lambda^{2}+\lambda \mu+\mu^{2}\right)^{2}} & \frac{(\lambda+1) \mu^{3}-\left(\lambda-\lambda^{2}\right) \mu^{2}-\left(\lambda^{2}-\lambda^{3}\right) \mu}{\left(\lambda^{2}+\lambda \mu+\mu^{2}\right)^{2}} & \frac{\mu^{2}\left(\lambda^{2}+\lambda \mu-2 \lambda-2 \mu+\mu^{2}\right)}{\left(\lambda^{2}+\lambda \mu+\mu^{2}\right)^{2}} \\
\frac{\lambda(\lambda+1) \mu^{2}-\lambda\left(\lambda-\lambda^{2}\right) \mu-\lambda\left(\lambda^{2}-\lambda^{3}\right)}{\left(\lambda^{2}+\lambda \mu+\mu^{2}\right)^{2}} & \frac{\lambda^{3} \mu+\lambda^{3}+\lambda^{2} \mu^{2}+\mu^{3}(\lambda+1)}{\left(\lambda^{2}+\lambda \mu+\mu^{2}\right)^{2}} & \frac{\mu(1+\mu) \lambda^{2}-\mu\left(\mu-\mu^{2}\right) \lambda-\mu\left(\mu^{2}-\mu^{3}\right)}{\left(\lambda^{2}+\lambda \mu+\mu^{2}\right)^{2}} \\
\frac{\lambda^{2}\left(\lambda^{2}+\lambda \mu-2 \lambda+\mu^{2}-2 \mu\right)}{\left(\lambda^{2}+\lambda \mu+\mu^{2}\right)^{2}} & \frac{(1+\mu) \lambda^{3}-\left(\mu-\mu^{2}\right) \lambda^{2}-\left(\mu^{2}-\mu^{3}\right) \lambda}{\left(\lambda^{2}+\lambda \mu+\mu^{2}\right)^{2}} & \frac{\lambda^{2}\left(\mu^{2}+3 \mu\right)+\lambda\left(\mu^{3}+\mu^{2}\right)+\lambda^{3}+\mu^{4}}{\left(\lambda^{2}+\lambda \mu+\mu^{2}\right)^{2}}
\end{array}\right) .
\end{aligned}
$$

In order to investigate the accuracy of the approximation developed in Section 2.2, we compare the obtained numerical results with those obtained by Monte Carlo simulation and analytical results. Note that the analytical expression used to calculate the expectation and variance of the continuous random variable $\pi(\lambda(\omega))$ is given by

$$
\mathbb{E}[\pi(\lambda(\omega))]=\int_{-\infty}^{+\infty} \pi(\lambda) f_{\lambda}(\lambda(\omega)) \mathrm{d} \lambda, \quad \operatorname{Var}(\pi(\lambda(\omega)))=\int_{-\infty}^{+\infty}(\pi(r)-\mathbb{E}[\pi(\lambda(\omega))])^{2} f_{\lambda}(r) \mathrm{d} r
$$

where $f_{\lambda}$ is the probability density function of $\lambda(\omega)$.

In our numerical experiments, all calculations are done with Matlab Software Package. For the numerical experiments, we let $\xi=10^{-3}, \mu=2, \bar{\lambda}=3$ and $\sigma=0.4$. Then, applying (3.1)-(3.4), we obtain

$$
\pi_{\bar{\lambda}}=(0.4737,0.3158,0.2105), Q_{\bar{\lambda}}=\left(\begin{array}{ccc}
-2 & 2 & 0 \\
2 & -5 & 2 \\
0 & 3 & -3
\end{array}\right) \text {, and } Z_{\bar{\lambda}}=\left(\begin{array}{ccc}
0.6454 & 0.2548 & 0.0997 \\
0.3823 & 0.4127 & 0.2050 \\
0.2244 & 0.3075 & 0.4681
\end{array}\right) .
$$

In this case, the obtained value of the bound of the remainder term, by applying (2.22), is 0.0060 and the degree of the Taylor polynomial required for achieving this precision is $k=4$. Moreover, Condition (C) is satisfied. In fact, we obtain $c=0.1163<1.5625$, and the radius of convergence $r$ obtained for the same values of parameters is 1.9000 . Note that condition $(2.24)$ is satisfied, i.e., $0.5263<1.2500$. The behavior of the upper bound for the expected value of the remainder term with respect to changes of the order of Taylor series expansions is shown in Figure 2. The expected value of the remainder term for the precision $\xi=10^{-3}$ is estimated at $\left\|\mathbb{E}\left[R_{\theta}(4, \varepsilon(\omega))\right]\right\|=3.0206 \times 10^{-4}$. 


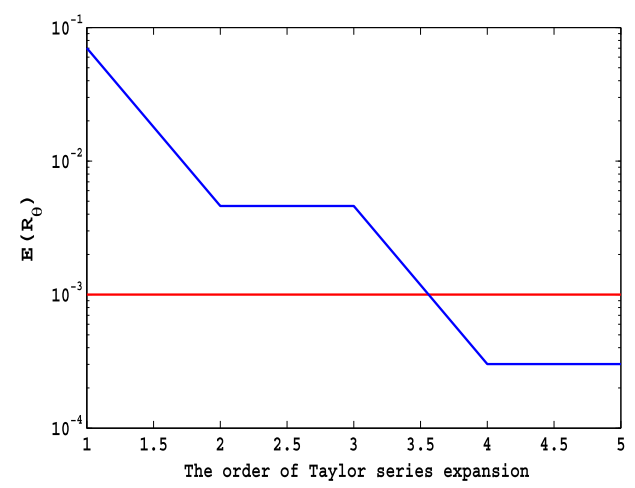

FiguRE 2. The upper bound for the expected value of the remainder term for the precision $\xi=10^{-3}$.

TABLE 1 . Expected value of $\pi_{i}, i=0,1,2$.

\begin{tabular}{llll}
\hline \hline Expected value & $\pi_{0}$ & $\pi_{1}$ & $\pi_{2}$ \\
\hline Taylor series expansion & 0.4703 & 0.3150 & 0.2147 \\
Monte Carlo simulation & 0.4711 & 0.3145 & 0.2144 \\
Analytical expectation & 0.4683 & 0.3120 & 0.2122 \\
\hline
\end{tabular}

TABLE 2. Variance of $\pi_{i}, i=0,1,2$

\begin{tabular}{llll}
\hline \hline Variance & $\pi_{0}$ & $\pi_{1}$ & $\pi_{2}$ \\
\hline Taylor series expansion & 0.0022 & 0.0001 & 0.0014 \\
Monte Carlo simulation & 0.0024 & 0.0002 & 0.0015 \\
Analytical variance & 0.0021 & 0.0001 & 0.0013 \\
\hline
\end{tabular}

We compare the expected value and the variance of each component of the stationary distribution $\pi(\omega)$ by using the three approaches (i) Taylor series expansion, (ii) Monte Carlo simulation and (iii) the analytical expression. The results are provided in Tables 1 and 2 .

We conclude with a risk analysis. Following the framework developed in Section 2.5. We choose the cost function $f$ as an indicator function on $i=0$, i.e.,

$$
f(i)= \begin{cases}1, & \text { if } i=0 \\ 0, & \text { otherwise }\end{cases}
$$

With this choice and using (2.30), we will apply the bound in (2.30) for the risk that $\pi_{0}(\lambda(\omega))$ deviates more than $r$ percent from the numerical value $\pi_{0}(\bar{\lambda})$. The Chebyshev's inequality bound put forward in (2.30) yields

$$
P\left(\left|\pi_{0}(\lambda(\omega))-\pi_{0}(\bar{\lambda})\right| \geq(0.01 \times r) \times \pi_{0}(\bar{\lambda})\right) \leq \frac{\operatorname{Var}\left(\pi_{0}(\lambda(\omega))\right)}{\left(\frac{r}{100} \pi_{0}(\bar{\lambda})-\left|\mathbb{E}\left[\pi_{0}(\lambda(\omega))\right]-\pi_{0}(\bar{\lambda})\right|\right)^{2}} .
$$

Numerical results shown in Figure 3. 


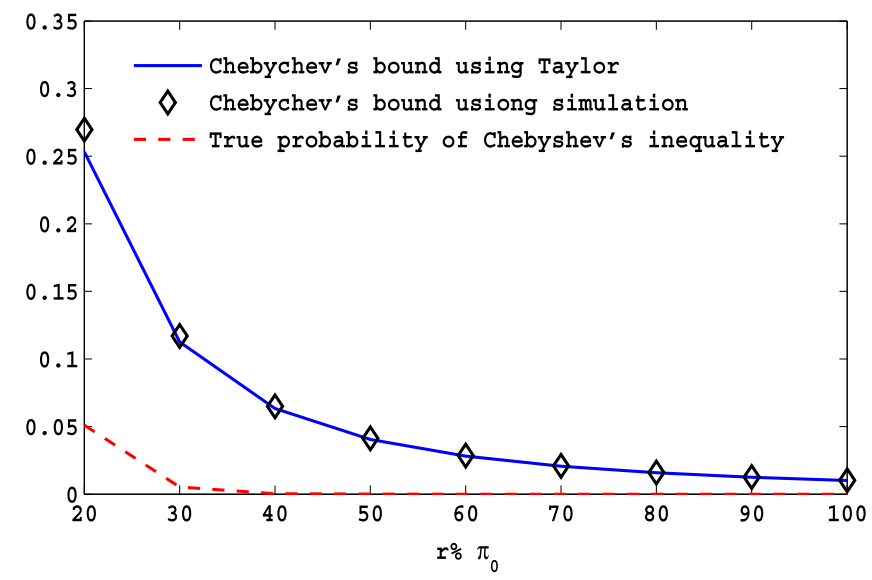

FigURE 3. Risk bounds versus true probability for: $\sigma=0.4, \bar{\lambda}=3, \mu=2$.

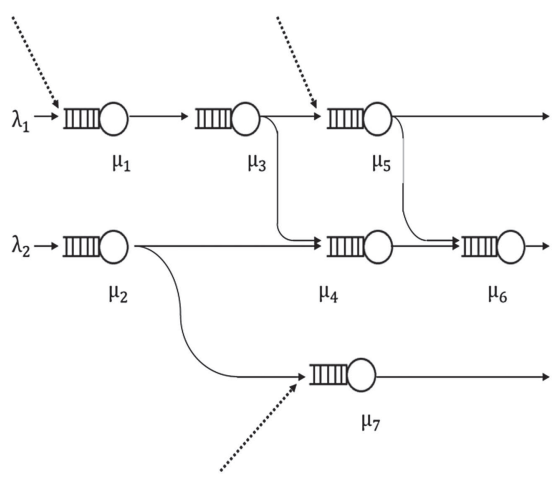

Figure 4. Queuing network example.

\subsection{Network with group breakdowns and repairs}

In this section we discuss an example taken from [32]. For details and background we refer to this reference. Consider the Jackson network with $J=7$ nodes (Fig. 4), where we assume throughout that servers are FCFS single-server stations with infinite waiting capacity, and let $\widetilde{J}=\{1, \ldots, 7\}$. We identify the labeling of the service rate $\mu_{i}$ with the server labels so that $\mu_{i}$ refers to server $i$. There are two arrival streams with arrival rate $\lambda_{1}$ and $\lambda_{2}$, respectively, and throughout the paper, it is assumed that arrival processes are of Poisson type. Routing is Markovian, and arrows indicate possible routes. Apart from these classical features of a Jackson network, we assume that there is a set of nodes $V=\{1,5,7\}$ that have infinite supply. For $j \in V$, customers in the infinite supply chain have low priority, where customers arriving either from the outside or from another server have high priority with the preemptive-resume regime: Service of a low priority customer is interrupted as soon as a high priority customer arrives. When a low priority customer is served and fed into the network, she/he becomes a high priority customer. Routing decisions, service times, and inter-arrival times are assumed to be mutually independent.

Suppose that $\lambda_{1}<\mu_{1}, \mu_{3}$ and $\mu_{1}>\mu_{3}$. Then, without infinite supply at node 1 , node 3 is stable in the classical definition as the rate with which customers arrive at node 3 is smaller than the service rate. In case of infinite supply at node 1 , however, node 1 acts as Poisson source and the incoming traffic rate at node 3, which is then $\mu_{1}$, is larger than the service rate, which causes node 3 to become unstable; for details see [32]. 
We consider the following possible breakdown scenarios $\emptyset,\{3\},\{5\},\{3,5\}$. We assume that the rate with which a breakdown of server $i=3,5$ occurs is given by $\tau_{i}$, and the corresponding repair rate by $\rho_{i}$. In the same vein, let $\tau_{3}+\tau_{5}$ be the rate with which the operating system enters breakdown state $\{3,5\}$, and let $2 \min \left(\rho_{3}, \rho_{5}\right)$ be the rate with which the systems jumps from breakdown state $\{3,5\}$ to the state $\emptyset$ with all server operating.

It is shown in [32] that the stationary distribution $\pi$ on the breakdown scenarios is given by

$$
\pi(\emptyset)=c^{-1}, \pi(\{3\})=c^{-1} \quad \frac{\tau_{3}}{\rho_{3}}, \pi(\{5\})=c^{-1} \quad \frac{\tau_{5}}{\rho_{5}}, \pi(\{3,5\})=c^{-1} \frac{\tau_{3}+\tau_{5}}{2 \min \left(\rho_{3}, \rho_{5}\right)}
$$

with normalizing constant

$$
c=1+\frac{\tau_{3}}{\rho_{3}}+\frac{\tau_{5}}{\rho_{5}}+\frac{\tau_{3}+\tau_{5}}{2 \min \left(\rho_{3}, \rho_{5}\right)} .
$$

\subsubsection{Analysis of the system performance}

Initially, we assume that the exact value of the rate $\tau_{3}$ is not well known, hence this parameter is called the uncertain parameter and it is supposed random variable with known mean and variance. This random variable is then written using the following linear perturbation

$$
\tau_{3}(\omega)=\bar{\tau}_{3}+\sigma \varepsilon(\omega),
$$

where $\bar{\tau}_{3}, \sigma$, are respectively estimated mean and the standard deviation of the random variable $\tau_{3}(\omega), \varepsilon(\omega)$ is the random variable modeling the parametric uncertainty on the parameter $\tau_{3}$.

Taking into account the uncertainty in the parameter $\tau_{3}$, the stationary vector $\pi_{\tau_{3}}$, on the set of states $\{\emptyset,\{3\},\{5\},\{3,5\}\}$ is viewed as the output function of the Jackson network model. We let $\theta=\tau_{3}$ and write $\pi_{\tau_{3}}^{(n)}$ for the $n$th derivative of a function $\pi_{\theta}$ with respect to $\theta$ evaluated at $\theta=\bar{\tau}_{3}$. Letting $Q_{\tau_{3}}$ denote the generator matrix of the breakdown process and $Z_{\tau_{3}}$ the corresponding fundamental matrix, we have from Remark 2.3 that

$$
\frac{1}{n !} \pi_{\bar{\tau}_{3}}^{(n)}=\pi_{\bar{\tau}_{3}}\left(Q_{\bar{\tau}_{3}}^{\prime} Z_{\bar{\tau}_{3}}\right)^{n}
$$

for $n \geq 1$. Under the assumption that $\varepsilon(\omega)$ is normal distributed, the approximation of the expectation and variance in closed-form expression provided in (2.14) and (2.15) become

$$
\mathbb{E}[\pi(\omega)]=\sum_{p=0}^{\infty} \sigma^{2 p} \pi_{\bar{\tau}_{3}}\left(Q_{\bar{\tau}_{3}}^{\prime} Z_{\bar{\tau}_{3}}\right)^{2 p}(2 p-1) ! !
$$

and

$$
\begin{aligned}
\operatorname{Var}\left[\pi_{i}(\omega)\right]= & \sum_{n=1}^{\infty} \sum_{m=1}^{\infty} \alpha_{i, n} \alpha_{i, m} \operatorname{Cov}\left(\varepsilon(\omega)^{n}, \varepsilon(\omega)^{m}\right) \\
= & \sum_{p=1}^{\infty} \sum_{q=1}^{\infty} \alpha_{i, 2 p} \alpha_{i, 2 q}[(2 p+2 q-1) ! !-(2 p-1) ! !(2 q-1) ! !] \\
& +\sum_{p=0}^{\infty} \sum_{q=0}^{\infty} \alpha_{i, 2 p+1} \alpha_{i, 2 q+1}(2 p+2 q+1) ! !
\end{aligned}
$$

where

$$
\alpha_{i, k}=\frac{\sigma^{k}}{k !} \pi_{\bar{\tau}_{3}}^{(k)}
$$

Tables 3 and 4 provide the numerical results for the expected value and variance of the stationary vector via Taylor series expansion and the simulation. Here, the parameters of the Jackson model are fixed as follows: 
TABLE 3. Expected value of the stationary distribution $\pi$.

\begin{tabular}{lllll}
\hline \hline Expected value & $\pi_{\emptyset}$ & $\pi_{\{3\}}$ & $\pi_{\{5\}}$ & $\pi_{\{3,5\}}$ \\
\hline Taylor series expansion & 0.2829 & 0.2303 & 0.2122 & 0.2743 \\
Monte Carlo simulation & 0.2828 & 0.2305 & 0.2121 & 0.2743 \\
Analytical expectation & 0.2830 & 0.2303 & 0.2122 & 0.2743 \\
\hline
\end{tabular}

TABLE 4. Variance of the stationary distribution $\pi$.

\begin{tabular}{lllll}
\hline \hline Variance & $\pi_{\emptyset}$ & $\pi_{\{3\}}$ & $\pi_{\{5\}}$ & $\pi_{\{3,5\}}$ \\
\hline Taylor series expansion $10^{-2}$ & 0.2194 & 0.5216 & 0.1234 & 0.0095 \\
Monte Carlo simulation $10^{-2}$ & 0.2270 & 0.5396 & 0.1277 & 0.0098 \\
Analytical variance $10^{-2}$ & 0.2274 & 0.5406 & 0.1279 & 0.0098 \\
\hline
\end{tabular}

$\tau_{5}=0.9, \rho_{5}=1.2, \rho_{3}=0.8$, whereas, the uncertain rate with which a breakdown of server $i=3$ occurs is normally distributed with mean $\bar{\tau}_{3}=0.7$ and standard deviation $\sigma=0.3$.

Let $f$ the cost function defined as

$$
f(i)= \begin{cases}1, & \text { if } i=\emptyset \\ 0, & \text { otherwise. }\end{cases}
$$

The risk in taking $\pi_{\emptyset}\left(\bar{\tau}_{3}\right)$ as output measure rather than $\pi_{\emptyset}(\omega)$ is given by

$$
\pi_{\emptyset}(\omega)-\pi_{\emptyset}\left(\bar{\tau}_{3}\right) .
$$

To assess the risk incurred by working with $\pi_{\emptyset}(\omega)$ one wants to bound

$$
P\left(\left|\pi_{\emptyset}(\omega)-\pi_{\emptyset}\left(\bar{\tau}_{3}\right)\right| \geq(0.01 \times r) \times \pi_{\emptyset}\left(\bar{\tau}_{3}\right)\right) .
$$

So, using the Chebyshev's inequality (2.30), this risk can be bounded by the following inequality

$$
P\left(\left|\pi_{\emptyset}(\omega)-\pi_{\emptyset}\left(\bar{\tau}_{3}\right)\right| \geq(0.01 \times r) \times \pi_{\emptyset}\left(\bar{\tau}_{3}\right)\right) \leq \frac{\operatorname{Var}\left(\pi_{\emptyset}(\omega)\right)}{\left(\frac{r}{100} \pi_{\emptyset}\left(\bar{\tau}_{3}\right)-\left|\mathbb{E}\left[\pi_{\emptyset}(\omega)\right]-\pi_{\emptyset}\left(\bar{\tau}_{3}\right)\right|\right)^{2}} .
$$

The numerical results of the computation of the bound is summarized in Figure 5 for the first component of the stationary distribution.

\section{The multiple-PARAmeter CASE}

\subsection{Higher-order sensitivities}

A formula appropriate for the higher-order sensitivity of the continuous-time Markov chain stationary distribution with respect to multiple-parameter follows from a development similar to that of the previous section. To this end, we let $\theta=\left(\theta_{1}, \theta_{2}, \ldots, \theta_{m}\right)$. Let us now assume that the continuous-time Markov chain generator matrix $Q$ depends on $m$ parameters, $\theta_{1}, \theta_{2}, \ldots, \theta_{m}$, in writing $Q_{\theta}=Q\left(\theta_{1}, \theta_{2}, \ldots, \theta_{m}\right)$. We will denote its stationary distribution by $\pi_{\theta}=\pi\left(\theta_{1}, \theta_{2}, \ldots, \theta_{m}\right)$. In the sequel, we are typically interested in establishing a closed-form expression for the higher-order sensitivity of stationary distribution to multiple-parameter, which is a multivariate analogue to that in Theorem 2.1. In this regard, we assume that all components of the generator matrix $Q_{\theta}$ are differentiable functions with respect to each parameter $\theta_{i}, 1 \leq i \leq m$. In order to provide a compact way 


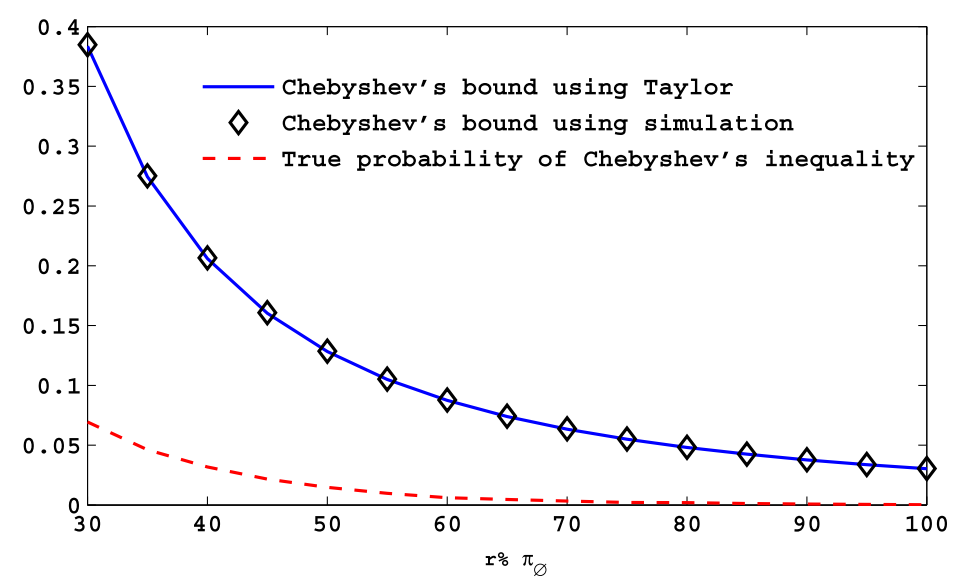

FigURE 5. Risk bounds versus true probability for the component $\pi_{\{\emptyset\}}$.

to establish the Taylor expansion of the stationary distribution $\pi_{\theta}$, we introduce the following notation of the multi-index $\imath=\left(\imath_{1}, \imath_{2}, \ldots, \imath_{m}\right)$, which is an $m$-tuple of nonnegative integers

$$
|\imath|=\imath_{1}+\imath_{2}+\ldots+\imath_{m}, \quad \imath !=\imath_{1} ! \times \imath_{2} ! \times \ldots \times \imath_{m} !, \quad \theta^{\imath}=\theta_{1}^{\imath_{1}} \times \theta_{2}^{\imath_{2}} \times \ldots \times \theta_{m}^{\imath_{m}}
$$

for $\imath \in \mathbb{N}^{m}$ and $\theta \in \mathbb{R}^{m}$. If $\kappa=\left(\kappa_{1}, \kappa_{2}, \ldots, \kappa_{m}\right)$ is another multi-index, then the sum and difference of $\imath$ and $\kappa$ is defined component-wise as

$$
\imath \pm \kappa=\left(\imath_{1} \pm \kappa_{1}, \imath_{2} \pm \kappa_{2}, \ldots, \imath_{m} \pm \kappa_{m}\right) .
$$

For multi-indices $\imath, \kappa$, with $\kappa \leq \imath$ (i.e., $\kappa_{p} \leq \imath_{p}$ for all $\left.p=1, \ldots, m\right)$, we define

$$
\left(\begin{array}{c}
\imath \\
\kappa
\end{array}\right)=\left(\begin{array}{c}
\imath_{1} \\
\kappa_{1}
\end{array}\right)\left(\begin{array}{c}
\imath_{2} \\
\kappa_{2}
\end{array}\right) \cdots\left(\begin{array}{c}
\imath_{m} \\
\kappa_{m}
\end{array}\right) \text {. }
$$

Similarly, if the $|\imath|$ th order partial derivative of $\pi_{\theta}$ exist in some neighborhood of $\theta$, then these ones are denoted by

$$
D^{\imath} \pi_{\theta}=\frac{\partial^{|\imath|} \pi_{\theta}}{\partial \theta_{1}^{\imath_{1}} \partial \theta_{2}^{\imath_{2}} \ldots \partial \theta_{m}^{\imath_{m}}} .
$$

In the following, we state the main result concerning the higher-order derivatives of the stationary distribution with respect to multiple-parameter for finite continuous-time Markov chains. We assume that $Q_{\theta}$ is infinitely often element-wise differentiable and denote the matrix of element-wise $n$th partial derivatives with respect to $\theta_{i}$ by

$$
\frac{\partial Q_{\theta}}{\partial \theta_{i}}
$$

The precise statement concerning the multivariate version of the higher-order derivatives of the stationary distribution $\pi_{\theta}$ with respect to $\theta=\left(\theta_{1}, \theta_{2}, \ldots, \theta_{m}\right)$ are given, in terms of the fundamental matrix $Z_{\theta}$, in the Theorem below.

Theorem 4.1. Let $\theta \in \Theta \subset \mathbb{R}^{m}$. Suppose that $Q_{\theta}$ is element-wise partially differentiable with respect to $\theta_{i}$, $1 \leq i \leq m$. Moreover, assume that all the $|\imath|$ th order partial derivatives are continuous with respect to $\theta$, then $\pi_{\theta}$ is $|\imath|$-times differentiable at the point $\theta$, and the $|\imath|$ th order partial derivatives of the stationary distribution $\pi_{\theta}$ are given by

$$
D^{\imath} \pi_{\theta}=\sum_{|\kappa|<|\imath|}\left(\begin{array}{c}
2 \\
\kappa
\end{array}\right) D^{\kappa} \pi_{\theta} D^{\imath-\kappa} Q_{\theta} Z_{\theta}
$$

where $\kappa$ and $\imath$ are two multi-indices. 
Proof. We prove the theorem by induction and in order to simplify the proof we consider $m=2$, i.e., $\theta=\left(\theta_{1}, \theta_{2}\right)$. For that, introduce the following statement

$$
\left(\mathcal{S}_{\mathbf{n}}\right): \frac{\partial^{|\imath|} \pi_{\theta}}{\partial \theta_{1}^{\imath_{1}} \partial \theta_{2}^{\imath_{2}}}=\sum_{|\kappa|<|\imath|}\left(\begin{array}{c}
\imath_{1} \\
\kappa_{1}
\end{array}\right)\left(\begin{array}{c}
\imath_{2} \\
\kappa_{2}
\end{array}\right) \frac{\partial^{|\kappa|} \pi_{\theta}}{\partial \theta_{1}^{\kappa_{1}} \partial \theta_{2}^{\kappa_{2}}}\left(\frac{\partial^{|\imath-\kappa|} Q_{\theta}}{\partial \theta_{1}^{\imath_{1}-\kappa_{1}} \partial \theta_{2}^{\iota_{2}-\kappa_{2}}}\right) Z_{\theta} .
$$

The initial step of the proof consists to prove that the statement $\left(\mathcal{S}_{\mathbf{n}}\right)$ is true for $|\imath|=2$. For $\theta_{1} \neq \theta_{2}$, we will show that

$$
\frac{\partial^{2} \pi_{\theta}}{\partial \theta_{1} \partial \theta_{2}}=\frac{\partial \pi_{\theta}}{\partial \theta_{1}} \frac{\partial Q_{\theta}}{\partial \theta_{2}} Z_{\theta}+\frac{\partial \pi_{\theta}}{\partial \theta_{2}} \frac{\partial Q_{\theta}}{\partial \theta_{1}} Z_{\theta}+\pi_{\theta} \frac{\partial^{2} Q_{\theta}}{\partial \theta_{1} \partial \theta_{2}} Z_{\theta}
$$

We have

$$
\left\{\begin{array}{l}
\pi_{\left(\theta_{1}, \theta_{2}\right)} Q_{\left(\theta_{1}, \theta_{2}\right)}=0 \\
\pi_{\left(\theta_{1}, \theta_{2}\right)} e=1
\end{array}\right.
$$

Taking the derivative of both sides of the equations (4.3) with respect to $\theta_{1}$, we obtain

$$
\left\{\begin{array}{l}
\frac{\partial \pi_{\theta}}{\partial \theta_{1}} Q_{\theta}+\pi_{\theta} \frac{\partial Q_{\theta}}{\partial \theta_{1}}=0 \\
\frac{\partial \pi_{\theta}}{\partial \theta_{1}} e=0
\end{array}\right.
$$

Again taking the derivative of both sides of the equations (4.4) with respect to $\theta_{2}$ yields that

$$
\left\{\begin{array}{l}
\frac{\partial^{2} \pi_{\theta}}{\partial \theta_{1} \partial \theta_{2}} Q_{\theta}+\frac{\partial \pi_{\theta}}{\partial \theta_{1}} \frac{\partial Q_{\theta}}{\partial \theta_{2}}+\frac{\partial \pi_{\theta}}{\partial \theta_{2}} \frac{\partial Q_{\theta}}{\partial \theta_{1}}+\pi_{\theta} \frac{\partial^{2} Q_{\theta}}{\partial \theta_{1} \partial \theta_{2}}=0 \\
\frac{\partial^{2} \pi_{\theta}}{\partial \theta_{1} \partial \theta_{2}} e=0
\end{array}\right.
$$

Following the same line of argument in (2.5), one obtains

$$
\frac{\partial^{2} \pi_{\theta}}{\partial \theta_{1} \partial \theta_{2}} \Pi_{\theta}=0
$$

Subtracting the equation (4.5) from the first equation of (4.4), we arrive at

$$
\frac{\partial^{2} \pi_{\theta}}{\partial \theta_{1} \partial \theta_{2}} Q_{\theta}-\underbrace{\frac{\partial^{2} \pi_{\theta}}{\partial \theta_{1} \partial \theta_{2}} \Pi_{\theta}}_{=0}+\frac{\partial \pi_{\theta}}{\partial \theta_{1}} \frac{\partial Q_{\theta}}{\partial \theta_{2}}+\frac{\partial \pi_{\theta}}{\partial \theta_{2}} \frac{\partial Q_{\theta}}{\partial \theta_{1}}+\pi_{\theta} \frac{\partial^{2} Q_{\theta}}{\partial \theta_{1} \partial \theta_{2}}=0 .
$$

It follows from simple algebra that $\left(\Pi_{\theta}-Q_{\theta}\right)$ is invertible. Hence, (4.2) holds.

Using the fact that all the $\imath$ th order partial derivatives of $\pi_{\theta}$ are continuous with respect to $\theta$, then one can change the order of mixed derivatives at $\theta$, so that we obtain

$$
\frac{\partial^{2} \pi_{\theta}}{\partial \theta_{1} \partial \theta_{2}}=\frac{\partial^{2} \pi_{\theta}}{\partial \theta_{2} \partial \theta_{1}}
$$

For $\theta_{1}=\theta_{2}$, we will obtain the same result stated in Theorem 2.1.

Secondly, we prove that if the statement $\left(\mathcal{S}_{\mathbf{n}}\right)$ is true for $|\imath|=k$, then it must also be true for $|\imath|=k+1$. So, we have

$$
\frac{\partial^{|\imath|} \pi_{\theta}}{\partial \theta_{1}^{\imath_{1}} \partial \theta_{2}^{\imath_{2}}}=\sum_{|\kappa|<|\imath|}\left(\begin{array}{c}
\imath_{1} \\
\kappa_{1}
\end{array}\right)\left(\begin{array}{c}
\imath_{2} \\
\kappa_{2}
\end{array}\right) \frac{\partial^{|\kappa|} \pi_{\theta}}{\partial \theta_{1}^{\kappa_{1}} \partial \theta_{2}^{\kappa_{2}}}\left(\frac{\partial^{|\imath-\kappa|} Q_{\theta}}{\partial \theta_{1}^{\imath_{1}-\kappa_{1}} \partial \theta_{2}^{\imath_{2}-\kappa_{2}}}\right) Z_{\theta} .
$$


Multiplying both sides of the equation (4.6) on the right by $\left(\Pi_{\theta}-Q_{\theta}\right)$, we obtain

$$
\frac{\partial^{|\imath|} \pi_{\theta}}{\partial \theta_{1}^{\imath_{1}} \partial \theta_{2}^{\imath_{2}}}\left(\Pi_{\theta}-Q_{\theta}\right)=\sum_{|\kappa|<|\imath|}\left(\begin{array}{c}
\imath_{1} \\
\kappa_{1}
\end{array}\right)\left(\begin{array}{c}
\imath_{2} \\
\kappa_{2}
\end{array}\right) \frac{\partial^{|\kappa|} \pi_{\theta}}{\partial \theta_{1}^{\kappa_{1}} \partial \theta_{2}^{\kappa_{2}}}\left(\frac{\partial^{|\imath-\kappa|} Q_{\theta}}{\partial \theta_{1}^{\imath_{1}-\kappa_{1}} \partial \theta_{2}^{\imath_{2}-\kappa_{2}}}\right) .
$$

Taking into account that

This yields

$$
\frac{\partial^{|\imath|} \pi_{\theta}}{\partial \theta_{1}^{\ell_{1}} \partial \theta_{2}^{\imath_{2}}} \Pi_{\theta}=0
$$

$$
-\frac{\partial^{|\imath|} \pi_{\theta}}{\partial \theta_{1}^{\imath_{1}} \partial \theta_{2}^{\imath_{2}}} Q_{\theta}=\sum_{|\kappa|<|\imath|}\left(\begin{array}{c}
\imath_{1} \\
\kappa_{1}
\end{array}\right)\left(\begin{array}{c}
\imath_{2} \\
\kappa_{2}
\end{array}\right) \frac{\partial^{|\kappa|} \pi_{\theta}}{\partial \theta_{1}^{\kappa_{1}} \partial \theta_{2}^{\kappa_{2}}}\left(\frac{\partial^{|\imath-\kappa|} Q_{\theta}}{\partial \theta_{1}^{\imath_{1}-\kappa_{1}} \partial \theta_{2}^{\imath_{2}-\kappa_{2}}}\right) .
$$

Taking derivatives of both sides of equation (4.7) with respect to $\theta_{1}$, then we obtain

$$
-\frac{\partial^{|\imath|+1} \pi_{\theta}}{\partial \theta_{1}^{\imath_{1}+1} \partial \theta_{2}^{\imath_{2}}} Q_{\theta}-\frac{\partial^{|\imath|} \pi_{\theta}}{\partial \theta_{1}^{\imath_{1}} \partial \theta_{2}^{\iota_{2}}} \frac{\partial Q_{\theta}}{\partial \theta_{1}}=S_{1}+S_{2}
$$

where

$$
S_{1}=\sum_{|\kappa|<|\imath|}\left(\begin{array}{c}
\imath_{1} \\
\kappa_{1}
\end{array}\right)\left(\begin{array}{c}
\imath_{2} \\
\kappa_{2}
\end{array}\right) \frac{\partial^{|\kappa|+1} \pi_{\theta}}{\partial \theta_{1}^{\kappa_{1}+1} \partial \theta_{2}^{\kappa_{2}}}\left(\frac{\partial^{|\imath-\kappa|} Q_{\theta}}{\partial \theta_{1}^{\imath_{1}-\kappa_{1}} \partial \theta_{2}^{2_{2}-\kappa_{2}}}\right),
$$

and

$$
S_{2}=\sum_{|\kappa|<|\imath|}\left(\begin{array}{c}
\imath_{1} \\
\kappa_{1}
\end{array}\right)\left(\begin{array}{c}
\imath_{2} \\
\kappa_{2}
\end{array}\right) \frac{\partial^{|\kappa|} \pi_{\theta}}{\partial \theta_{1}^{\kappa_{1}} \partial \theta_{2}^{\kappa_{2}}}\left(\frac{\partial^{|\imath-\kappa|+1} Q_{\theta}}{\partial \theta_{1}^{\imath_{1}+1-\kappa_{1}} \partial \theta_{2}^{\imath_{2}-\kappa_{2}}}\right) .
$$

After some algebra and using the fact that

$$
\left(\begin{array}{c}
\imath_{1} \\
\kappa_{1}
\end{array}\right)+\left(\begin{array}{c}
\imath_{1} \\
\kappa_{1}-1
\end{array}\right)=\left(\begin{array}{c}
\imath_{1}+1 \\
\kappa_{1}
\end{array}\right)
$$

we arrive at

$$
-\frac{\partial^{|\imath|+1} \pi_{\theta}}{\partial \theta_{1}^{\imath_{1}+1} \partial \theta_{2}^{\imath_{2}}} Q_{\theta}=\sum_{|\kappa|<|\imath|+1}\left(\begin{array}{c}
\imath_{1}+1 \\
\kappa_{1}
\end{array}\right)\left(\begin{array}{c}
\iota_{2} \\
\kappa_{2}
\end{array}\right) \frac{\partial^{|\kappa|} \pi_{\theta}}{\partial \theta_{1}^{\kappa_{1}} \partial \theta_{2}^{\kappa_{2}}}\left(\frac{\partial^{|\imath-\kappa|+1} Q_{\theta}}{\partial \theta_{1}^{\imath_{1}+1-\kappa_{1}} \partial \theta_{2}^{2_{2}-\kappa_{2}}}\right) .
$$

Inserting $\frac{\partial^{|2|+1} \pi_{\theta}}{\partial \theta_{1}^{\nu_{1}+1} \partial \theta_{2}^{22}} \Pi_{\theta}$, which equal to zero, on the left-hand side of equation (4.8). By simple algebra, we obtain

$$
\frac{\partial^{|\imath|+1} \pi_{\theta}}{\partial \theta_{1}^{\imath_{1}+1} \partial \theta_{2}^{\imath_{2}}}=\sum_{|\kappa|<|\imath|+1}\left(\begin{array}{c}
\imath_{1}+1 \\
\kappa_{1}
\end{array}\right)\left(\begin{array}{c}
\iota_{2} \\
\kappa_{2}
\end{array}\right) \frac{\partial^{|\kappa|} \pi_{\theta}}{\partial \theta_{1}^{\kappa_{1}} \partial \theta_{2}^{\kappa_{2}}}\left(\frac{\partial^{|\imath-\kappa|+1} Q_{\theta}}{\partial \theta_{1}^{\imath_{1}+1-\kappa_{1}} \partial \theta_{2}^{2_{2}-\kappa_{2}}}\right) Z_{\theta},
$$

which concludes the proof of the statement.

Remark 4.2. As has already been mentioned in the previous case, in case that the components of the generator matrix $Q_{\theta}$ are affine linear with respect to each $\theta_{i}, i=1, \ldots, m$, we obtain for all $k \geq 2$

$$
\frac{\partial^{k} Q_{\theta}}{\partial \theta_{i}^{k}}=0
$$

Introduce the following finite multiset $I$ of ordered indices

$$
I=\{\underbrace{1, \ldots, 1}_{\imath_{1}}, \underbrace{2, \ldots, 2}_{\imath_{2}}, \ldots, \underbrace{m, \ldots, m}_{\imath_{m}}\}
$$


where each element $p$ appears exactly as often as is its multiplicity $\imath_{p}$ in $I$. Let $A_{p} \subseteq I$ be a distinguishable permutation of elements of $I$, each multiset $A_{p}$ has exactly $|\imath|$ ordered indices, then the total number of distinguishable permutations in $I$ is

$$
\varpi=\left(\begin{array}{c}
|\imath| \\
\imath_{1}, \ldots, \imath_{m}
\end{array}\right)=\frac{|\imath| !}{\imath_{1} ! \ldots \imath_{m} !},
$$

which yields $A_{1}, \ldots, A_{\varpi}$ multiset permutations.

Thereby, we get the equivalent version of (4.1) for the case of linear perturbation, which is given by

$$
\frac{\partial^{|\imath|} \pi_{\bar{\theta}}}{\partial \theta_{1}^{\imath_{1}} \ldots \partial \theta_{m}^{i_{m}}}=\imath ! \pi_{\theta} \sum_{p=1}^{\varpi} \prod_{j \in A_{p}}\left(\frac{\partial Q_{\theta}}{\partial \theta_{j}} Z_{\theta}\right)
$$

Furthermore, to the best of our knowledge, the new formula (4.9) has not been introduced previously in the literature. This constitutes a generalization of the earlier works $[6,8,9,11]$, as it addresses the question whether establishing a closed-form expression for higher-order sensitivity of the continuous-time Markov chain stationary distribution to multiple-parameter, which is derived in terms of the fundamental matrix.

Example 4.3. For cost function $f, \pi_{\theta} f$ is totally differentiable. Its derivative is given by the gradient, which has elements

$$
\frac{\partial}{\partial \theta_{i}} \pi_{\theta} f=\pi_{\theta}\left(\frac{\partial Q_{\theta}}{\partial \theta_{i}} Z_{\theta}\right)
$$

and the elements of the Hessian of $\pi_{\theta} f$ are given by

$$
\frac{\partial^{2}}{\partial \theta_{i} \partial \theta_{j}} \pi_{\theta} f=\pi_{\theta}\left(\frac{\partial Q_{\theta}}{\partial \theta_{i}} Z_{\theta}\right)\left(\frac{\partial Q_{\theta}}{\partial \theta_{j}} Z_{\theta}\right)+\pi_{\theta}\left(\frac{\partial Q_{\theta}}{\partial \theta_{j}} Z_{\theta}\right)\left(\frac{\partial Q_{\theta}}{\partial \theta_{i}} Z_{\theta}\right)
$$

where $I=\{i, j\}, \varpi=2$, so two permutations in $I$ are possible: $A_{1}=\{i, j\}$, and $A_{2}=\{j, i\}$ for $i \neq j$, and for $i=j$, we get

$$
\frac{\partial^{2}}{\partial \theta_{i}^{2}} \pi_{\theta} f=2 ! \pi_{\theta}\left(\frac{\partial Q_{\theta}}{\partial \theta_{i}} Z_{\theta}\right)^{2}
$$

\subsection{Statistical Taylor series expansions: Multiple-parameters}

In this section, we discuss the multivariate Taylor series expansion for propagating the uncertainty in continuous-time Markov chain stationary distribution, due to epistemic uncertainties in the model input parameters. More precisely, using the higher-order sensitivity of the stationary distribution with respect to multiple parameters, which is introduced in (4.9), we propose an approximate method based on Taylor series expansion for computing the expected value and the variance of the stationary distribution $\pi(\omega)$, which is function of random variables: $\theta_{1}(\omega), \ldots, \theta_{m}(\omega)$. In this sense, under the conditions of Theorem 4.1, the Taylor series expansion of the stationary distribution $\pi(\omega)$ of multiple-parameter $\theta(\omega)=\left(\theta_{1}(\omega), \ldots, \theta_{m}(\omega)\right)$, can be written compactly as

$$
\pi(\omega) \triangleq \pi_{\bar{\theta}+\sigma \varepsilon(\omega)}=\sum_{|\imath| \geq 0} \frac{(\theta(\omega)-\bar{\theta})^{\imath}}{\imath !} D^{\imath} \pi_{\theta} .
$$


More explicitly

$$
\begin{aligned}
\pi_{\bar{\theta}+\sigma \varepsilon(\omega)}= & \sum_{i_{1}=0}^{\infty} \ldots \sum_{i_{m}=0}^{\infty} \frac{\left(\theta_{1}(\omega)-\bar{\theta}_{1}\right)^{i_{1}} \ldots\left(\theta_{m}(\omega)-\bar{\theta}_{m}\right)^{i_{m}}}{i_{1} ! \ldots i_{m} !}\left(\frac{\partial^{i_{1}+\ldots+i_{m}} \pi}{\partial \theta_{1}^{i_{1}} \ldots \partial \theta_{m}^{i_{m}}}\right)\left(\theta_{1}, \ldots, \theta_{m}\right) \\
= & \pi\left(\bar{\theta}_{1}, \ldots, \bar{\theta}_{m}\right)+\sum_{j=1}^{m} \frac{\partial \pi\left(\theta_{1}, \ldots, \theta_{m}\right)}{\partial \theta_{j}}\left(\theta_{j}(\omega)-\bar{\theta}_{j}\right) \\
& +\frac{1}{2 !} \sum_{j=1}^{m} \sum_{k=1}^{m} \frac{\partial^{2} \pi\left(\theta_{1}, \ldots, \theta_{m}\right)}{\partial \theta_{j} \partial \theta_{k}}\left(\theta_{j}(\omega)-\bar{\theta}_{j}\right)\left(\theta_{k}(\omega)-\bar{\theta}_{k}\right) \\
& +\frac{1}{3 !} \sum_{j=1}^{m} \sum_{k=1}^{m} \sum_{l=1}^{m} \frac{\partial^{3} \pi\left(\theta_{1}, \ldots, \theta_{m}\right)}{\partial \theta_{j} \partial \theta_{k} \partial \theta_{l}}\left(\theta_{j}(\omega)-\bar{\theta}_{j}\right)\left(\theta_{k}(\omega)-\bar{\theta}_{k}\right)\left(\theta_{l}(\omega)-\bar{\theta}_{l}\right)+\ldots
\end{aligned}
$$

Note that for each parameter $\theta_{i}, 1 \leq i \leq m$, we associate a model under the form (1.1), i.e., for all $i$, such that $1 \leq i \leq m$, we let

$$
\theta_{i}(\omega)=\bar{\theta}_{i}+\sigma_{i} \varepsilon_{i}(\omega)
$$

where $\bar{\theta}_{i}$ and $\sigma_{i}$ represent the estimated mean value and the standard deviation associated with the random variable $\theta_{i}(\omega)$, respectively, and $\varepsilon_{i}(\omega)$ is a zero mean normal random variable, modeling the epistemic uncertainty distribution.

Example 4.4. The Taylor series to third order around the point $\left(\bar{\theta}_{1}, \bar{\theta}_{2}\right)$ is given as follows

$$
\begin{aligned}
\pi\left(\bar{\theta}_{1}+\sigma_{1} \varepsilon_{1}, \bar{\theta}_{2}+\sigma_{2} \varepsilon_{2}\right)= & \pi\left(\bar{\theta}_{1}, \bar{\theta}_{2}\right)+\frac{\partial \pi}{\partial \theta_{1}} \sigma_{1} \varepsilon_{1}+\frac{\partial \pi}{\partial \theta_{2}} \sigma_{2} \varepsilon_{2} \\
& +\frac{1}{2}\left[\frac{\partial^{2} \pi}{\partial \theta_{1}^{2}} \sigma_{1}^{2} \varepsilon_{1}^{2}+2 \frac{\partial^{2} \pi}{\partial \theta_{1} \partial \theta_{2}} \sigma_{1} \varepsilon_{1} \sigma_{2} \varepsilon_{2}+\frac{\partial^{2} \pi}{\partial \theta_{2}^{2}} \sigma_{2}^{2} \varepsilon_{2}^{2}\right] \\
& +\frac{1}{6}\left[\frac{\partial^{3} \pi}{\partial \theta_{1}^{3}} \sigma_{1}^{3} \varepsilon_{1}^{3}+3 \frac{\partial^{3} \pi}{\partial \theta_{1}^{2} \partial \theta_{2}} \sigma_{1}^{2} \varepsilon_{1}^{2} \sigma_{2} \varepsilon_{2}+3 \frac{\partial^{3} \pi}{\partial \theta_{1} \partial \theta_{2}^{2}} \sigma_{1} \varepsilon_{1} \sigma_{2}^{2} \varepsilon_{2}^{2}+\frac{\partial^{3} \pi}{\partial \theta_{2}^{3}} \sigma_{2}^{3} \varepsilon_{2}^{3}\right] \\
& +R\left(3, \varepsilon_{1}(\omega), \varepsilon_{2}(\omega)\right),
\end{aligned}
$$

where $R\left(3, \varepsilon_{1}(\omega), \varepsilon_{2}(\omega)\right)$ is the Taylor series remainder.

Taking into account the linear form of the higher-order derivatives of the stationary distribution $\pi_{\theta}$ with respect to $\theta=\left(\theta_{1}, \theta_{2}, \ldots, \theta_{m}\right)$, that are given in (4.9), the expression of the Taylor series expansion introduced above in (4.11) can be rewritten as

$$
\begin{aligned}
\pi_{\bar{\theta}+\sigma \varepsilon(\omega)} & =\sum_{|\imath| \geq 0} \frac{(\theta(\omega)-\bar{\theta})^{\imath}}{\imath !} \frac{\partial^{|\imath|} \pi_{\bar{\theta}}}{\partial \theta_{1}^{\imath_{1}} \ldots \partial \theta_{m}^{z_{m}}} \\
& =\pi_{\bar{\theta}} \sum_{|\imath| \geq 0} \sum_{p=1}^{\varpi} \prod_{j \in A_{p}}\left(\left(\theta_{j}(\omega)-\bar{\theta}_{j}\right) \frac{\partial Q_{\bar{\theta}}}{\partial \theta_{j}} Z_{\bar{\theta}}\right),
\end{aligned}
$$

where $\bar{\theta}=\left(\bar{\theta}_{1}, \ldots, \bar{\theta}_{m}\right)$.

Thereby, based on the Taylor expansion approach introduced here and assuming that the (mixed) moments $\mathbb{E}\left[(\varepsilon(\omega))^{\imath}\right]$ are finite, the expected value and the variance of the stationary distribution $\pi(\omega)$ can be easily numerically computed through the following formulae

$$
\mathbb{E}[\pi(\omega)]=\sum_{|\imath| \geq 0} \frac{D^{\imath} \pi_{\theta}}{\imath !} \mathbb{E}\left[(\theta(\omega)-\bar{\theta})^{\imath}\right]=\sum_{|\imath| \geq 0} \sigma^{\imath} \frac{D^{\imath} \pi_{\theta}}{\imath !} \mathbb{E}\left[(\varepsilon(\omega))^{\imath}\right],
$$


where $\sigma^{\imath}=\prod_{p=1}^{m} \sigma_{p}^{\imath_{p}}=\sigma_{1}^{\imath_{1}} \ldots \sigma_{m}^{\imath_{m}}$ and $(\varepsilon(\omega))^{\imath}=\prod_{p=1}^{m}\left(\varepsilon_{p}(\omega)\right)^{\imath_{p}}=\left(\varepsilon_{1}(\omega)\right)^{\imath_{1}} \ldots\left(\varepsilon_{m}(\omega)\right)^{\imath_{m}}$.

It is obvious from the above equations that it is required that the (mixed) moments $\mathbb{E}\left[(\varepsilon(\omega))^{\imath}\right]$ are finite. In the following we discuss the impact of possible dependence of $\left(\epsilon_{i}(\omega), 1 \leq i \leq m\right)$.

\subsubsection{Independent input parameters}

We assume that the random variables $\varepsilon_{p}^{\imath_{p}}(\omega)$ are independent for all $p, 1 \leq p \leq m$. Therefore, the expected value of $\pi(\omega)$ in (4.14) can be explicitly computed as follows

$$
\begin{aligned}
\mathbb{E}[\pi(\omega)] & =\sum_{|\imath| \geq 0} \frac{D^{\imath} \pi_{\theta}}{\imath !} \mathbb{E}\left[(\theta(\omega)-\bar{\theta})^{\imath}\right] \\
& =\sum_{|\imath| \geq 0} \sigma^{\imath} \frac{D^{\imath} \pi_{\theta}}{\imath !} \mathbb{E}\left[\prod_{p=1}^{m}\left(\varepsilon_{p}(\omega)\right)^{\imath_{p}}\right] \\
& =\sum_{|\imath| \geq 0} \sigma^{\imath} \frac{D^{\imath} \pi_{\theta}}{\imath !} \prod_{p=1}^{m} \mathbb{E}\left[\left(\varepsilon_{p}(\omega)\right)^{\imath_{p}}\right] \\
& =\sum_{|\jmath| \geq 0} \sigma^{2 \jmath} \frac{D^{(2 \jmath)} \pi_{\theta}}{(2 \jmath) !} \prod_{p=1}^{m}\left(2 \jmath_{p}-1\right) ! !
\end{aligned}
$$

For a truncated Taylor series, the expected value is given as follows

$$
\mathbb{E}[\pi(\omega)]=\pi_{\bar{\theta}} \sum_{|\jmath| \geq 0}^{\lceil k / 2\rceil}(2 \jmath-1) ! ! \sum_{h=1}^{\varpi} \prod_{l \in B_{h}}\left(\frac{\partial Q_{\bar{\theta}}}{\partial \theta_{l}} Z_{\bar{\theta}} \sigma_{l}\right),
$$

where

$$
\varpi=\frac{|2 \jmath| !}{\left(2 \jmath_{1}\right) ! \ldots\left(2 \jmath_{m}\right) !},
$$

and $B_{h}$ is a multiset permutation of elements of

$$
I=\{\underbrace{1, \ldots, 1}_{2 \jmath_{1}}, \underbrace{2, \ldots, 2}_{2 \jmath_{2}}, \ldots, \underbrace{m, \ldots, m}_{2 \jmath_{m}}\}
$$

Example 4.5. The approximate expected value of the stationary distribution $\pi\left(\theta_{1}, \theta_{2}\right)$ is given by the following formula

$$
\begin{aligned}
\mathbb{E}\left[\pi\left(\theta_{1}, \theta_{2}\right)\right] & \approx \pi_{\bar{\theta}} \sum_{\jmath_{1}=0}^{1} \sum_{\jmath_{2}=0}^{1}\left(2 \jmath_{1}-1\right) ! !\left(2 \jmath_{2}-1\right) ! ! \sum_{h=1}^{\varpi} \prod_{l \in \mathbf{B}_{h}}\left(\frac{\partial Q_{\bar{\theta}}}{\partial \theta_{l}} Z_{\bar{\theta}} \sigma_{l}\right) \\
& =\pi_{\bar{\theta}}+(2-1) ! ! \pi_{\bar{\theta}} \prod_{l \in\{1,1\}}\left(\frac{\partial Q_{\bar{\theta}}}{\partial \theta_{l}} Z_{\bar{\theta}} \sigma_{l}\right)+(2-1) ! ! \pi_{\bar{\theta}} \prod_{l \in\{2,2\}}\left(\frac{\partial Q_{\bar{\theta}}}{\partial \theta_{l}} Z_{\bar{\theta}} \sigma_{l}\right) \\
& =\pi_{\bar{\theta}}+\pi_{\bar{\theta}}\left(\frac{\partial Q_{\bar{\theta}}}{\partial \theta_{1}} Z_{\bar{\theta}} \sigma_{1}\right)^{2}+\pi_{\bar{\theta}}\left(\frac{\partial Q_{\bar{\theta}}}{\partial \theta_{2}} Z_{\bar{\theta}} \sigma_{2}\right)^{2} .
\end{aligned}
$$


Similarly, one can obtain an explicit expression of the variance of the stationary distribution $\pi_{i}(\omega)$. This is given by

$$
\begin{aligned}
\operatorname{Var}\left(\pi_{i}(\omega)\right) & =\sum_{|\imath| \geq 0}\left(\beta_{i, \imath}\right)^{2} \mathbb{V a r}\left(\prod_{p=1}^{m}\left(\varepsilon_{p}(\omega)^{\iota_{p}}\right)\right) \\
& =\sum_{|\imath| \geq 0}\left(\beta_{i, \imath}\right)^{2}\left[\mathbb{E}\left[\prod_{p=1}^{m} \varepsilon_{p}(\omega)^{2 \imath_{p}}\right]-\left[\mathbb{E} \prod_{p=1}^{m}\left(\varepsilon_{p}(\omega)^{\imath_{p}}\right)\right]^{2}\right] \\
& =\sum_{|\imath| \geq 0}\left(\beta_{i, \imath}\right)^{2}\left[\prod_{p=1}^{m} \mathbb{E}\left(\varepsilon_{p}(\omega)^{2 \imath_{p}}\right)-\prod_{p=1}^{m}\left[\mathbb{E}\left(\varepsilon_{p}(\omega)^{\imath_{p}}\right)\right]^{2}\right] \\
& =\sum_{|\imath| \geq 0}\left(\beta_{i, \imath}\right)^{2} \prod_{p=1}^{m} \mathbb{E}\left(\varepsilon_{p}(\omega)^{2 \imath_{p}}\right)-\sum_{|\ell| \geq 0}\left(\beta_{i, 2 \ell}\right)^{2} \prod_{p=1}^{m}\left[\mathbb{E}\left(\varepsilon_{p}(\omega)^{2 \ell_{p}}\right)\right]^{2} \\
& =\sum_{|\imath| \geq 0}\left(\beta_{i, \imath}\right)^{2} \prod_{p=1}^{m}\left(2 \imath_{p}-1\right) ! !-\sum_{|\ell| \geq 0}\left(\beta_{i, 2 \ell}\right)^{2} \prod_{p=1}^{m}\left[\left(2 \ell_{p}-1\right) ! !\right]^{2}
\end{aligned}
$$

where

$$
\beta_{i, \imath}=\sigma^{\imath} \frac{D^{\imath} \pi_{\theta}}{\imath !} e_{i}^{T}
$$

and

$$
e_{i}^{T}=(0 \ldots, \underbrace{1}_{\text {at the } i \text { position }}, \ldots 0)
$$

\subsubsection{Dependent input parameters}

Assume that for all nonnegative integers $p$, such that $1 \leq p \leq m$, the random variables $\varepsilon_{p}(\omega)$ are dependent with correlation matrix

$$
\Sigma=\left(\operatorname{Cov}\left(\epsilon_{j}, \epsilon_{k}\right)\right)_{1 \leq j \leq m ; 1 \leq k \leq m},
$$

commonly called "the covariance matrix". In this regard, we discuss a general way of computing the expected value and the variance of $\pi(\omega)$, where the mixed moments $\mathbb{E}\left[\prod_{p=1}^{m}\left(\varepsilon_{p}(\omega)\right)^{\imath_{p}}\right]$ are expressed in terms of covariance matrix $\Sigma$. That allows us to study the effect correlation between the random variables $\varepsilon_{p}(\omega), 1 \leq p \leq m$, has. If, for example, we suppose that $\varepsilon_{p}(\omega)$ follows the standard normal distribution, and $\Sigma$ is $m \times m$ positive semidefinite matrix. Then the expected value of the stationary distribution $\pi(\omega)$ is given by

$$
\mathbb{E}[\pi(\omega)]=\sum_{|\imath| \geq 0} \sigma^{\imath} \frac{D^{\imath} \pi_{\theta}}{\imath !} \mathbb{E}\left[\prod_{p=1}^{m}\left(\varepsilon_{p}(\omega)\right)^{\imath_{p}}\right] .
$$

Explicit expressions of the expectation $\mathbb{E}\left[\prod_{p=1}^{m}\left(\varepsilon_{p}(\omega)\right)^{\imath_{p}}\right]$ have long been available in the statistics literature $[3,15,18,19,25,26,31,35]$. We will use the following elegant formula obtained in [19]:

$$
\mathbb{E}\left[\prod_{p=1}^{m}\left(\varepsilon_{p}(\omega)\right)^{\imath_{p}}\right]= \begin{cases}\frac{1}{(|\imath| / 2) !} \sum_{\nu=0}^{\imath}(-1)^{|\nu|}\left(\begin{array}{c}
\imath \\
\nu
\end{array}\right)\left(\frac{h^{T} \Sigma h}{2}\right)^{\imath / 2}, & \text { if }|\imath| \text { is even } \\
0, & \text { if }|\imath| \text { is odd }\end{cases}
$$

where $h=\left(\imath_{1} / 2-\nu_{1}, \ldots, \iota_{m} / 2-\nu_{m}\right)^{T}, \nu=\left(\nu_{1}, \ldots, \nu_{m}\right)$, and $|\nu|=\nu_{1}+\ldots+\nu_{m}$. 
Inserting (4.18) into the truncated Taylor series, one can easily provide a compact closed form expression for the expectation of $\pi_{\theta}$ as follows

$$
\begin{aligned}
\mathbb{E}[\pi(\omega)]= & \sum_{|\jmath| \geq 0}^{\lceil k / 2\rceil} \sigma^{2 \jmath} \frac{D^{(2 \jmath)} \pi_{\theta}}{(2 \jmath) !} \frac{1}{|\jmath| !} \sum_{\nu=0}^{2 \jmath}(-1)^{|\nu|}\left(\begin{array}{c}
2 \jmath \\
\nu
\end{array}\right)\left(\frac{h_{1}^{T} \Sigma h_{1}}{2}\right)^{\jmath} \\
& +\sum_{|\ell| \geq 0}^{\lceil k / 2\rceil-1} \sigma^{2 \ell+1} \frac{D^{(2 \ell+1)} \pi_{\theta}}{(2 \ell+1) !} \frac{1}{(|2 \ell+1| / 2) !} \sum_{\nu=0}^{2 \ell+1}(-1)^{|\nu|}\left(\begin{array}{c}
2 \ell+1 \\
\nu
\end{array}\right)\left(\frac{h_{2}^{T} \Sigma h_{2}}{2}\right)^{(|2 \ell+1| / 2)}
\end{aligned}
$$

where $h_{1}=\left(\jmath_{1} / 2-\nu_{1}, \ldots, \jmath_{m} / 2-\nu_{m}\right)^{T}$, and $h_{2}=\left(\left(2 \ell_{1}+1\right) / 2-\nu_{1}, \ldots,\left(2 \ell_{m}+1\right) / 2-\nu_{m}\right)^{T}$.

Analogously, one can compute the variance of $\pi_{i}(\omega)$ by using the following compressed formula

$$
\begin{aligned}
\operatorname{Var}\left(\pi_{i}(\omega)\right) & =\sum_{|\imath| \geq 0} \sum_{|\jmath| \geq 0} \beta_{i, \imath} \beta_{i, \jmath} \operatorname{Cov}\left(\varepsilon(\omega)^{\imath}, \varepsilon(\omega)^{\jmath}\right) \\
& =\sum_{|\imath| \geq 0} \sum_{|\jmath| \geq 0} \beta_{i, \imath} \beta_{i, \jmath}\left[\mathbb{E}\left[(\varepsilon(\omega))^{\imath+\jmath}\right]-\mathbb{E}\left[(\varepsilon(\omega))^{\imath}\right] \mathbb{E}\left[(\varepsilon(\omega))^{\jmath}\right]\right],
\end{aligned}
$$

where

$$
\beta_{i, \imath}=\sigma^{\imath} \frac{D^{\imath} \pi_{\theta}}{\imath !} e_{i}^{T}
$$

and

$$
\operatorname{Cov}\left(\varepsilon(\omega)^{\imath}, \varepsilon(\omega)^{\jmath}\right)=\operatorname{Cov}\left(\prod_{p=1}^{m} \varepsilon_{p}^{\iota_{p}}(\omega), \prod_{p=1}^{m} \varepsilon_{p}^{\jmath_{p}}(\omega)\right) .
$$

At this point, we may turn to problem of bounding the remainder term. Therefore, the Taylor series expansion introduced in (4.10) can be rewritten as follows

$$
\pi(\omega) \triangleq \pi_{\bar{\theta}+\sigma \varepsilon(\omega)}=\underbrace{\sum_{|q| \leq k} \frac{D^{\imath} \pi_{\theta}}{\imath !}(\theta(\omega)-\bar{\theta})^{\imath}}_{=T_{\theta}(k, \varepsilon(\omega))}+\underbrace{\sum_{|\imath| \geq k+1} \phi_{\imath}(\theta(\omega))(\theta(\omega)-\bar{\theta})^{\imath}}_{=R_{\theta}(k, \varepsilon(\omega))},
$$

where

$$
\lim _{\theta(\omega) \rightarrow \bar{\theta}} \phi_{\imath}(\theta(\omega))=0 .
$$

\subsection{Bounds on the statistical remainder term}

In this section, bounds for the remainder term are provided for the case of independent input parameters in Section 4.3.1, and for dependent parameters in Section 4.3.2.

\subsubsection{Independent input parameters case}

To bound the remainder term of the Taylor series expansion introduced in (4.21), we will follow the same line of arguments in [1]. Then, it follows that

$$
\begin{aligned}
\mathbb{E}\left[R_{\theta}(k, \varepsilon(\omega))\right] & =\sum_{|\imath| \geq k+1} \frac{D^{\imath} \pi_{\theta}}{\imath !} \mathbb{E}\left[(\theta(\omega)-\bar{\theta})^{\imath}\right] \\
& =\sum_{|\jmath| \geq\lceil k / 2\rceil+1} \sigma^{2 \jmath} \frac{D^{(2 \jmath)} \pi_{\theta}}{(2 \jmath) !} \prod_{p=1}^{m}\left(2 \jmath_{p}-1\right) ! !
\end{aligned}
$$


For computational purposes, we propose to truncate the above sum to some order $\kappa$. Therefore, we obtain the following estimation

$$
\mathbb{E}\left[R_{\theta}(k, \varepsilon(\omega))\right] \approx \sum_{|\jmath|=\lceil k / 2\rceil+1}^{u} \sigma^{2 \jmath} \frac{D^{(2 \jmath)} \pi_{\theta}}{(2 \jmath) !} \prod_{p=1}^{m}\left(2 \jmath_{p}-1\right) ! ! .
$$

Consider the following statement:

$(\mathfrak{C})$ There exists a finite constant $\delta$ such that

$$
\left\|D^{(2 \jmath)} \pi_{\theta}\right\| \leq \delta
$$

Then, we can obtain

$$
\begin{aligned}
\left\|\mathbb{E}\left[R_{\theta}(k, \varepsilon(\omega))\right]\right\| & \leq \sum_{|\jmath|=\lceil k / 2\rceil+1}^{u} \sigma^{2 \jmath} \frac{\delta}{(2 \jmath) !} \prod_{p=1}^{m}\left(2 \jmath_{p}-1\right) ! ! \\
& \leq \sum_{|\jmath|=\lceil k / 2\rceil+1}^{u} \sigma^{2 \jmath} \frac{\delta}{(2 \jmath) !} \prod_{p=1}^{m} \frac{\left(2 \jmath_{p}\right) !}{2^{\jmath_{p}} \jmath_{p} !} \\
& \leq \delta \sum_{|\jmath|=\lceil k / 2\rceil+1}^{u} \frac{\sigma^{2 \jmath}}{2^{\jmath} \jmath !} .
\end{aligned}
$$

\subsubsection{Dependent input parameters case}

Subsequently, we will establish an alternative estimation for the expectation of the remainder term in the case of the dependent input parameters. So, we have

$$
\begin{aligned}
\mathbb{E}\left[R_{\theta}(k, \varepsilon(\omega))\right] & \approx \sum_{|\imath|=k+1}^{u} \frac{D^{\imath} \pi_{\theta}}{\imath !} \mathbb{E}\left[(\theta(\omega)-\bar{\theta})^{\imath}\right] \\
& =\sum_{|\jmath|=\lceil k / 2\rceil+1}^{u} \sigma^{2 \jmath} \frac{D^{(2 \jmath)} \pi_{\theta}}{(2 \jmath) !} \frac{1}{|\jmath| !} \sum_{\nu=0}^{2 \jmath}(-1)^{|\nu|}\left(\begin{array}{c}
2 \jmath \\
\nu
\end{array}\right)\left(\frac{h_{1}^{T} \sum h_{1}}{2}\right)^{\jmath} .
\end{aligned}
$$

Under the assumption $(\mathfrak{C})$, one can obtain

$$
\left\|\mathbb{E}\left[R_{\theta}(k, \varepsilon(\omega))\right]\right\| \leq \delta \sum_{|\jmath|=\lceil k / 2\rceil+1}^{u} \frac{\sigma^{2 \jmath}}{(2 \jmath) !} \frac{1}{|\jmath| !} \sum_{\nu=0}^{2 \jmath}(-1)^{|\nu|}\left(\begin{array}{c}
2 \jmath \\
\nu
\end{array}\right)\left(\frac{h_{1}^{T} \sum h_{1}}{2}\right)^{\jmath} .
$$

\section{MARKOVIAN RELIABILITY SYSTEM With PARALLEL COMPONENTS}

A parallel system is a configuration such that, as long as not all of the system components fail, the entire system works. In this section, we consider a simple system built with two independent components; see Figure 6 . This system is operating correctly if at least one of the components is functioning. Assume that each component has two possible states, functioning, denoted by 1 , and out of order, denoted by 0 . Therefore, the system as a whole has four states: $0:=(0,0), 1:=(0,1), 2:=(1,0)$, and $3:=(1,1)$. For example, $(1,0)$ means that the first component is functioning whereas the second one failed. Let $\lambda_{i}$ and $\mu_{i}$ denote the failure rate and repair rate of component $i$ for $i=1,2$, respectively. The generator of the Markov chain can be written as

$$
Q_{\left(\lambda_{1}, \lambda_{2}\right)}=\left(\begin{array}{cccc}
-\left(\mu_{1}+\mu_{2}\right) & \mu_{2} & \mu_{1} & 0 \\
\lambda_{2} & -\left(\mu_{1}+\lambda_{2}\right) & 0 & \mu_{1} \\
\lambda_{1} & 0 & -\left(\lambda_{1}+\mu_{2}\right) & \mu_{2} \\
0 & \lambda_{1} & \lambda_{2} & -\left(\lambda_{1}+\lambda_{2}\right)
\end{array}\right) .
$$




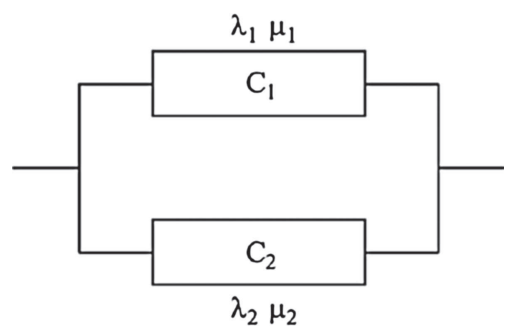

Figure 6. Parallel structure of two components.

Solving system in (2.3), we obtain

$$
\begin{aligned}
& \pi_{0}=\frac{\lambda_{1} \lambda_{2}}{\left(\lambda_{1}+\mu_{1}\right)\left(\lambda_{2}+\mu_{2}\right)}, \pi_{1}=\frac{\lambda_{1} \mu_{2}}{\left(\lambda_{1}+\mu_{1}\right)\left(\lambda_{2}+\mu_{2}\right)}, \\
& \pi_{2}=\frac{\lambda_{2} \mu_{1}}{\left(\lambda_{1}+\mu_{1}\right)\left(\lambda_{2}+\mu_{2}\right)}, \pi_{3}=\frac{\mu_{1} \mu_{2}}{\left(\lambda_{1}+\mu_{1}\right)\left(\lambda_{2}+\mu_{2}\right)},
\end{aligned}
$$

For our numerical purposes, we will compute the fundamental matrix $Z_{\left(\lambda_{1}, \lambda_{2}\right)}$ by matrix inversion using the fact that $Z_{\left(\lambda_{1}, \lambda_{2}\right)}=\left(\Pi_{\left(\lambda_{1}, \lambda_{2}\right)}-Q_{\left(\lambda_{1}, \lambda_{2}\right)}\right)^{-1}$.

To illustrate the applicability of the proposed approach outlined above in Section 6, we introduce two models for the following failure parameters

$$
\begin{array}{ll}
\lambda_{1}(\omega)=\bar{\lambda}_{1}+\sigma_{1} \varepsilon_{1}(\omega), & \varepsilon_{1} \rightsquigarrow \mathcal{N}(0,1), \\
\lambda_{2}(\omega)=\bar{\lambda}_{2}+\sigma_{2} \varepsilon_{2}(\omega), & \varepsilon_{2} \rightsquigarrow \mathcal{N}(0,1),
\end{array}
$$

where $\bar{\lambda}_{1}$ is the mean of the failure rate of the first component $\lambda_{1}$ and $\sigma_{1}$ is its standard deviation, $\bar{\lambda}_{2}$ is the mean of the failure rate of the second component $\lambda_{2}$ and $\sigma_{2}$ is its standard deviation, $\varepsilon_{1}$ the random variable modeling the epistemic uncertainty associated with $\lambda_{1}$ and $\varepsilon_{2}$ the random variable modeling the epistemic uncertainty inflicted on $\lambda_{2}$. Both random variables follow the standard normal distribution.

\subsection{Independent input parameters case}

Based on the results obtained in Section 4.2.1, we present some numerical examples. Without loss of generality, we assume that the failure rate of the first component $\lambda_{1}$ and the failure rate of the second component $\lambda_{2}$ are independent random variables. Throughout these numerical computations, we fix the following values of input parameters: $\mu_{1}=3, \mu_{2}=2, \overline{\lambda_{1}}=2.5, \sigma_{1}=0.3, \overline{\lambda_{2}}=4$ and $\sigma_{2}=0.5$.

For these fixed values of the input parameters, we obtain

$$
\begin{aligned}
\pi_{\left(\overline{\lambda_{1}}, \overline{\lambda_{2}}\right)}= & (0.3030,0.1515,0.3636,0.1818) \\
Q_{\left(\overline{\lambda_{1}}, \overline{\lambda_{2}}\right)}= & \left(\begin{array}{cccc}
-5.0000 & 2.0000 & 3.0000 & 0 \\
4.0000 & -7.0000 & 0 & 3.0000 \\
2.5000 & 0 & -4.5000 & 2.0000 \\
0 & 2.5000 & 4.0000 & -6.5000
\end{array}\right), \\
Z_{\left(\overline{\lambda_{1}}, \overline{\lambda_{2}}\right)}= & \left(\begin{array}{cccc}
0.4102 & 0.1435 & 0.3120 & 0.1343 \\
0.2870 & 0.2667 & 0.2685 & 0.1777 \\
0.2600 & 0.1119 & 0.4622 & 0.1659 \\
0.2238 & 0.1481 & 0.3318 & 0.2963
\end{array}\right)
\end{aligned}
$$

Let the precision be $\xi=10^{-5}$. Then, we will obtain the degree of Taylor polynomial $k=4$. Tables 5 and 6 summarize respectively the expected and the variance values of each component of the stationary distribution 
TABLE 5. Expected value of $\pi_{i}, i=0,1,2,3$.

\begin{tabular}{lllll}
\hline \hline Expected value & $\pi_{0}$ & $\pi_{1}$ & $\pi_{2}$ & $\pi_{3}$ \\
\hline Taylor series expansion & 0.3009 & 0.1520 & 0.3634 & 0.1837 \\
Monte Carlo simulation & 0.3014 & 0.1527 & 0.3623 & 0.1835 \\
Analytical expectation & 0.3001 & 0.1517 & 0.3625 & 0.1832 \\
\hline
\end{tabular}

TABLE 6 . Variance of $\pi_{i}, i=0,1,2,3$.

\begin{tabular}{lllll}
\hline \hline Variance & $\pi_{0} * 10^{-3}$ & $\pi_{1} * 10^{-3}$ & $\pi_{2} * 10^{-3}$ & $\pi_{3} * 10^{-3}$ \\
\hline Taylor series expansion & 0.5583 & 0.2614 & 0.6294 & 0.3326 \\
Monte Carlo simulation & 0.5170 & 0.2694 & 0.6150 & 0.3299 \\
Analytical variance & 0.5607 & 0.2663 & 0.6365 & 0.3420 \\
\hline
\end{tabular}

$\pi_{i}, i=0,1,2,3$, computed by the three approaches: Taylor series expansion, Monte Carlo simulation and the analytical one.

It may be observed from Tables 5 and 6 that the numerical values of the expected and the variance of each component of the stationary distribution $\pi$ evaluated through Taylor series expansion approach match precisely with the ones evaluated through the Monte Carlo simulation and the analytical approach.

\subsection{Dependent input parameters case}

In this subsection, we address the problem of the evaluation of the stationary distribution $\pi_{\left(\lambda_{1}, \lambda_{2}\right)}$, under the assumption that the uncertain input parameters $\lambda_{1}$ and $\lambda_{2}$ are dependent random variables. Using the Taylor series expansion introduced in Subsection 4.3.2, we consider the relationship between these two random variables. The dependence between $\lambda_{1}$ and $\lambda_{2}$ is expressed by the correlation $\rho$. The analytical expression used to calculate the expectation and variance are given by

$$
\begin{aligned}
\mathbb{E}\left[\pi\left(\lambda_{1}, \lambda_{2}\right)\right] & =\int_{-\infty}^{+\infty} \int_{-\infty}^{+\infty} \pi\left(\lambda_{1}, \lambda_{2}\right) f_{\lambda_{1}, \lambda_{2}}(\lambda(\omega)) \mathrm{d} \lambda_{1} \mathrm{~d} \lambda_{2} \\
\operatorname{Var}\left(\pi\left(\lambda_{1}, \lambda_{2}\right)\right) & =\int_{-\infty}^{+\infty} \int_{-\infty}^{+\infty}\left[\pi\left(\lambda_{1}, \lambda_{2}\right)-\mathbb{E}\left[\pi\left(\lambda_{1}, \lambda_{2}\right)\right)\right]^{2} f_{\lambda_{1}, \lambda_{2}}(\lambda(\omega)) \mathrm{d} \lambda_{1} \mathrm{~d} \lambda_{2},
\end{aligned}
$$

where the probability density function of $\lambda(\omega)$ is defined as follows

$$
\left.f_{\lambda_{1}, \lambda_{2}}(\lambda(\omega))=\frac{1}{2 \pi \sigma_{1} \sigma_{2} \sqrt{\left(1-\rho^{2}\right)}} \exp \left[-\frac{1}{2\left(1-\rho^{2}\right)}\left(\frac{\left(\Delta_{\lambda_{1}}\right)^{2}}{\sigma_{1}^{2}}\right)-\frac{2 \rho\left(\Delta_{\lambda_{1}}\right)\left(\Delta_{\lambda_{2}}\right)}{\sigma_{1} \sigma_{2}}+\frac{\left(\Delta_{\lambda_{2}}\right)^{2}}{\sigma_{2}^{2}}\right)\right],
$$

where $\left(\lambda_{i}-\bar{\lambda}_{i}\right)=\Delta_{\lambda_{i}}$. Note that the same computational scheme is considered above for the case where $\lambda_{1}$ and $\lambda_{2}$ are independent, with the following joint density function

$$
f_{\lambda_{1}, \lambda_{2}}(\lambda(\omega))=\frac{1}{2 \pi \sigma_{1} \sigma_{2}} \exp \left[-\frac{1}{2}\left(\frac{\left(\lambda_{1}-\bar{\lambda}_{1}\right)^{2}}{\sigma_{1}^{2}}+\frac{\left(\lambda_{2}-\bar{\lambda}_{2}\right)^{2}}{\sigma_{2}^{2}}\right)\right] .
$$

To perform the numerical analysis, we consider the same values of model parameters: $\mu_{1}=3, \mu_{2}=2, \overline{\lambda_{1}}=$ $2.5, \sigma_{1}=0.3, \overline{\lambda_{2}}=4$ and $\sigma_{2}=0.5$. Note that Condition $(\mathfrak{C})$ is satisfied for $\delta=0.06$. In the same vein, let $\xi=10^{-4}$, then we obtain the degree of Taylor polynomial $k=2$. 

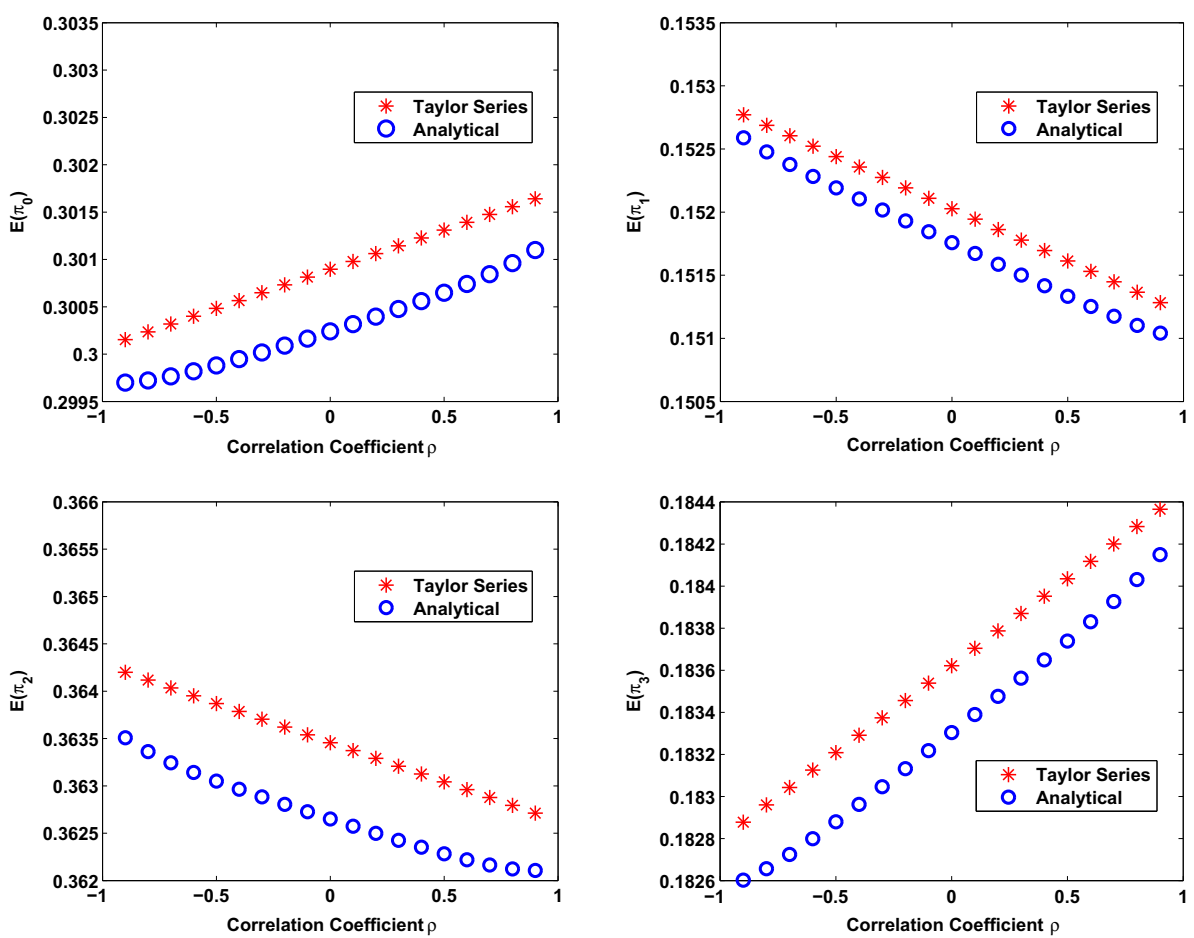

FIGURE 7. Expectation of the stationary distribution $\pi(\omega)$.

Certainly, the epistemic uncertainty inflicted in the failure rate parameters $\lambda_{1}$ and $\lambda_{2}$ may influence the stationary distribution $\pi_{\left(\lambda_{1}, \lambda_{2}\right)}$. So, we give a variety of numerical examples to explain such influence and the validity of obtained results. The graphs displayed in Figures 7 and 8 present the expectation and variance of each component of the stationary distribution $\pi_{i}, i=0,1,2,3$, computed numerically, by using the both approaches (Taylor series expansion and the analytical one), with variation of the correlation coefficient $\rho \in[-1,1]$.

It is to be noted that varying in the values of the correlation coefficient $\rho$, causes varying values of the expectation and variance of each component of the stationary distribution $\pi_{i}, i=0,1,2,3$, especially those corresponding to the variance of each component. The main insight provided by this example is that correlation $\rho$ effects the stationary state distributions for some states in a monotone increasing way whereas for others positive correlation leads to smaller stationary state distribution. These qualitative relations allow to judge the impact suspected correlation has on the performance metrics. For example, if we are interested in the stationary probability the system is fully functional, i.e., $\pi_{1}$, then we see that possible positive correlation will increase this probability but as the variance increases as well it is more risky to deduce the behavior of the fully functional state from $\pi\left(\bar{\lambda}_{1}, \bar{\lambda}_{2}\right)$. It is worth noting that this qualitative behavior is reversed for the stationary probabilities of states 1 and 2 .

\section{MARKOVIAN RELIABILITY SYSTEM OF AN $n$-UNIT STANDBY REPAIRABLE SYSTEM}

In this section, we consider a system that consists of $n$ identical units and $k$ repair facilities. At the beginning $(t=0)$, all units are good, and one unit is working and others are under cold standby. The operating unit fails after some time that has an exponential distribution with parameter $\lambda$. The repair facility will repair the working one as soon as it fails. At the same time, the standby one begins to work. The repair time is exponentially distributed with parameter $\mu$. Assume that each unit after repair is 'as good as new' and it either 

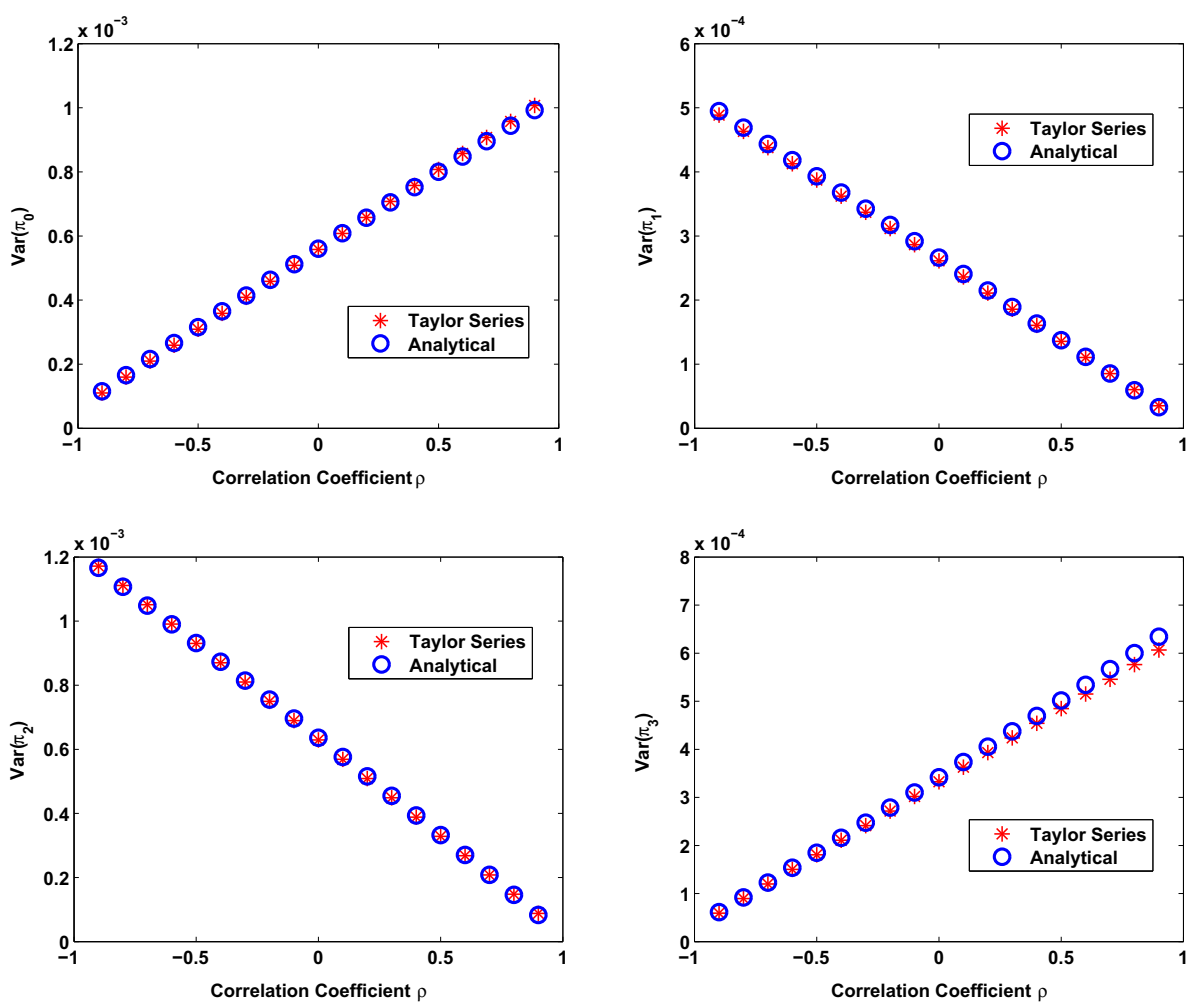

Figure 8. Variance of of the stationary distribution $\pi(\omega)$.

begins to work again or becomes under cold standby. If the rest units fail while $k$ units are still under repair, they must wait for repair. The switch is perfect, instantaneous. The random variables are supposed mutually independent. Let $x(t)$ denote the number of failed units (including the units that are under repair) at time $t$. The stochastic process $\{x(t), t \geq 0\}$ is Markov process with state space $E=\{0,1, \ldots, n\}$. The infinitesimal generator associated with the Markov process $\{x(t), t \geq 0\}$ is given by:

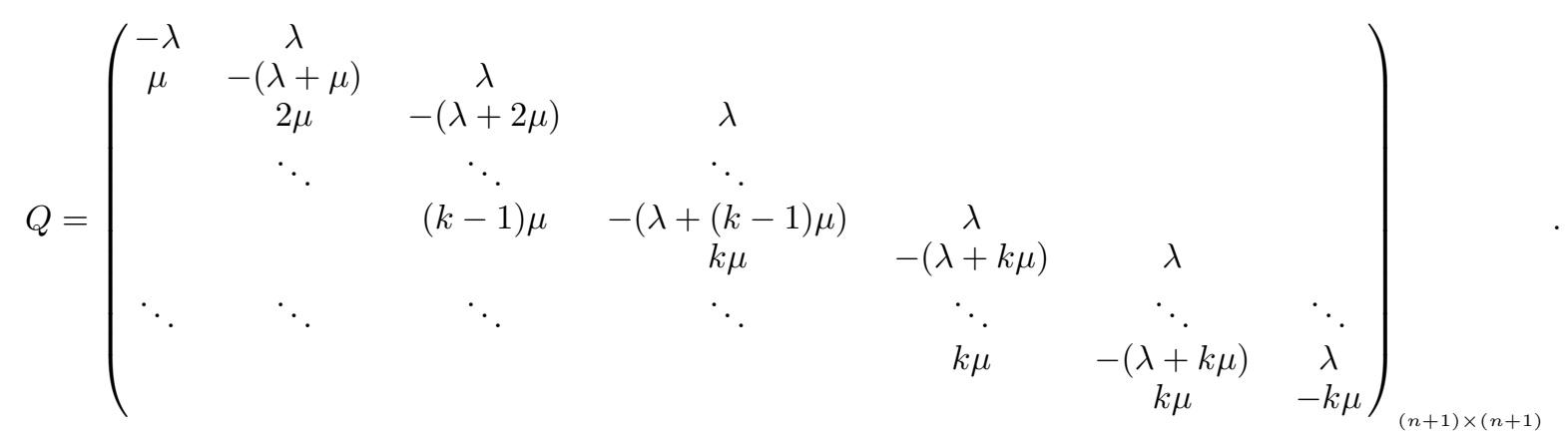

In the sequel, we assume that the failure rate $\lambda$ and repair rate $\mu$ are determined from a finite number of observations and has uncertainty associated with them. Using the approach outlined in Section, we will numerically calculate the expectation and the variance of some reliability indices deduced from the stationary distribution corresponding to the studied model. Particularly, the availability of the system, $A(\lambda, \mu)$, and failure frequency of the system, $f(\lambda, \mu)$. We assume that the uncertain input parameters $\lambda$ and $\mu$ are dependent random 

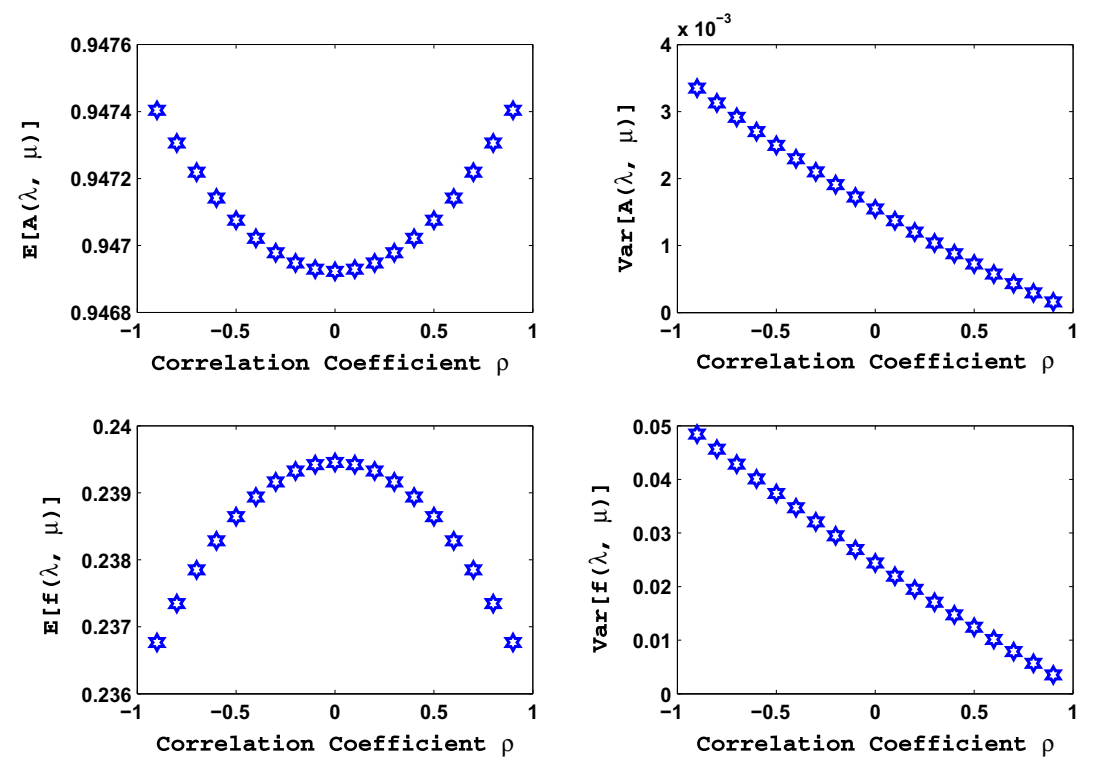

FIgURE 9. Statistical moments of the availability and failure frequency of the system using Taylor series expansions.

variables. To perform the numerical analysis, we introduce two models for the following parameters :

$$
\begin{array}{ll}
\lambda(\omega)=\bar{\lambda}+\sigma_{1} \varepsilon_{1}(\omega), & \varepsilon_{1} \rightsquigarrow \mathcal{N}(0,1), \\
\mu(\omega)=\bar{\mu}+\sigma_{2} \varepsilon_{2}(\omega), & \varepsilon_{2} \rightsquigarrow \mathcal{N}(0,1),
\end{array}
$$

where the input parameters are fixed as follows : $\bar{\lambda}=4, \sigma_{1}=0.5 \bar{\mu}=1$ and $\sigma_{2}=0.1$.

Let the precision be $\xi=10^{-4}$. Then, we will obtain the degree of Taylor polynomial $k=4$, and the estimation of the remainder term $\mathbb{E}\left[R_{\lambda, \mu}\left(k, \varepsilon_{1}(\omega), \varepsilon_{2}(\omega)\right)\right] \approx 9.2787 \times 10^{-6}$. The Figure 9 presents the expectation and variance of two indices, availability and failure frequency of a 10 unit standby repairable system with 5 repair facilities, computed numerically, by using the Taylor series expansion approach.

\section{Conclusion}

We have proposed a new method based on the statistical Taylor series expansion for finite continuous-time Markov chains with discrete state space. We have obtained a closed form expression of the higher derivatives of the stationary distribution in terms of the fundamental matrix associated with the Markov chain. These results were applied to compute the model output metrics of Markov reliability models, under propagation of epistemic uncertainty in the model input parameters. Specifically, an approximative expression for the expectation and variance of the components of stationary distribution of the Markov chain is established. We have also provided an expression for the remainder term of the Taylor series, and have included the effect of the correlation between the uncertain input parameters. In addition, we have estimated the risk incurred by working with uncertain performance measures rather than those evaluated at fixed parameter. Finally, we have performed some numerical examples to illustrate the potential of the proposed approach. The techniques adopted in this paper can be applied to analyze more complex models such as unreliable queueing models, which is left for future investigations. 
Acknowledgements. The authors thank the area editor and the referee for their valuable remarks and constructive comments that considerably improved the final version of this paper.

\section{REFERENCES}

[1] K. Abbas, B. Heidergott and D. Aïssani, A functional approximation for the $M / G / 1 / N$ queue. Discrete Event Dyn. Syst. 23 (2013) 93-104.

[2] J.L. Barlow, Stable computation with the fundamental matrix of a markov chain. SIAM J. Matrix Anal. App. 22 (2000) 230-241.

[3] R. Blacher, Multivariate quadratic forms of random vectors. J. Multivariate Anal. 87 (2003) 2-23.

[4] J.T. Blake, A.L. Reibman and K.S. Trivedi, Sensitivity analysis of reliability and performability measures for multiprocessor systems. In: Vol. 16 of ACM SIGMETRICS Performance Evaluation Review. ACM (1988) 177-186.

[5] P. Coolen-Schrijner and E.A. Van Doorn, The deviation matrix of a continuous-time markov chain. Probab. Eng. Inf. Sci. 16 (2002) 351-366.

[6] S.V. Dhople and A.D. Dominguez-Garcia, A parametric uncertainty analysis method for markov reliability and reward models. IEEE Trans. Reliab. 61 (2012) 634-648.

[7] A. Gandini, Importance and sensitivity analysis in assessing system reliability. IEEE Trans. Reliab. 39 (1990) 61-70.

[8] B.R. Haverkort and A.M. Meeuwissen, Sensitivity and uncertainty analysis in performability modelling. In: Proceedings 11th Symposium on Reliable Distributed Systems, 1992. IEEE (1992) 93-102.

[9] B.R. Haverkort and A.M.H. Meeuwissen, Sensitivity and uncertainty analysis of markov-reward models. IEEE Trans. Reliab. 44 (1995) 147-154.

[10] B. Heidergott and A. Hordijk, Taylor series expansions for stationary markov chains. Adv. Appl. Probab. 35 (2003) $1046-1070$.

[11] B. Heidergott, A. Hordijk and N. Leder, Series expansions for continuous-time markov processes. Oper. Res. 58 (2010) $756-767$.

[12] D.P. Heyman, Accurate computation of the fundamental matrix of a markov chain. SIAM J. Matrix Anal. App. 16 (1995) 954-963.

[13] D.P. Heyman and D.P. OLeary, What is fundamental for markov chains: first passage times, fundamental matrices, and group generalized inverses. In: Computations with Markov Chains. Springer (1995) 151-161.

[14] D.P. Heyman and A. Reeves, Numerical solution of linear equations arising in markov chain models. ORSA J. Comput. 1 (1989) 52-60.

[15] B. Holmquist, Moments and cumulants of the multivariate normal distribution. Stochastic Anal. App. 6 (1988) $273-278$.

[16] A. Hordijk and F. Spieksma, A new formula for the deviation matrix, chapter 36 of Probability Statistics and Optimization. Wiley (1994).

[17] J.J. Hunter, Generalized inverses and their application to applied probability problems. Linear Algebra App. 45 (1982) 157-198.

[18] L. Isserlis, On a formula for the product-moment coefficient of any order of a normal frequency distribution in any number of variables. Biometrika 12 (1918) 134-139.

[19] R. Kan, From moments of sum to moments of product. J. Multivariate Anal. 99 (2008) 542-554.

[20] J.G. Kemeny and J.L. Snell, Finite Markov Chains. D van Nostad Co. Inc., Princeton, NJ (1960).

[21] J.G. Kemeny and J.L. Snell, Finite continuous time markov chains. Theory Probab. App. 6 (1961) 101-105.

[22] S.J. Kirkland, M. Neumann and J. Xu, A divide and conquer approach to computing the mean first passage matrix for markov chains via perron complement reductions. Numer. Linear Algebra App. 8 (2001) 287-295.

[23] G.M. Koole and F.M. Spieksma, On deviation matrices for birth-death processes. Prob. Eng. Inf. Sci. 15 (2001) 239-258.

[24] I. Marek, Iterative aggregation/disaggregation methods for computing some characteristics of markov chains. II. Fast convergence. Appl. Numer. Math. 45 (2003) 11-28.

[25] J. Michalowicz, J. Nichols, F. Bucholtz and C. Olson, An isserlis theorem for mixed gaussian variables: application to the auto-bispectral density. J. Stat. Phys. 136 (2009) 89-102.

[26] J. Michalowicz, J. Nichols, F. Bucholtz and C. Olson, A general isserlis theorem for mixed-gaussian random variables. Stat. Probab. Lett. 81 (2011) 1233-1240.

[27] K. Mishra and K. Trivedi, A non-obtrusive method for uncertainty propagation in analytic dependability models. In: Proceedings of 4 th Asia-Pacific Symposium on Advanced Reliability and Maintenance Modeling (2010).

[28] S. Ouazine and K. Abbas, Development of computational algorithm for multiserver queue with renewal input and synchronous vacation. Appl. Math. Modell. 40 (2016) 1137-1156.

[29] P. Pukite and J. Pukite, Modeling for Reliability Analysis: Markov Modeling for Reliability, Maintainability, Safety, and Supportability. Wiley (1998).

[30] R.A. Sahner, K.S. Trivedi and A. Puliafito, Performance and Reliability Analysis of Computer Systems: An Example-based Approach Using the SHARPE Software Package. Springer Science \& Business Media (1996).

[31] J.R. Schott, Kronecker product permutation matrices and their application to moment matrices of the normal distribution. J. Multivariate Anal. 87 (2003) 177-190.

[32] J. Sommer, J. Berkhout, H. Daduna and B. Heidergott, Analysis of jackson networks with infinite supply and unreliable nodes. Queue. Syst. 87 (2017) 181-207.

[33] I. Sonin and J. Thornton, Recursive algorithm for the fundamental/group inverse matrix of a markov chain from an explicit formula. SIAM J. Matrix Anal. App. 23 (2001) 209-224. 
[34] K. Takaragi, R. Sasaki and S. Shingai, A probability bound estimation method in markov reliability analysis. IEEE Trans. Reliab. 34 (1985) 257-261.

[35] C. Vignat, A generalized isserlis theorem for location mixtures of gaussian random vectors. Stat. Probab. Lett. 82 (2012) 67-71.

[36] L. Yin, M.A. Smith and K.S. Trivedi, Uncertainty analysis in reliability modeling. In: Proceedings Annual Reliability and Maintainability Symposium, 2001. IEEE (2001) 229-234. 